\title{
Estimación del riesgo aguas abajo de una presa en función de las políticas de operación a largo plazo: caso de estudio presa El Cuchillo
}

\author{
Estimation of optimal operation policies associated with \\ the generated affectations: case study El Cuchillo dam
}

\author{
Sadoth Fabián Huerta Loera ${ }^{1 *} \quad$ Ramón Domínguez Mora ${ }^{2}$ \\ Recibido 6 de mayo de 2015, aceptado 9 de mayo de 2016 \\ Received: Mayo 6, 2015 Accepted: May 9, 2016
}

\begin{abstract}
RESUMEN
La práctica común para evaluar el riesgo de inundaciones aguas abajo de una presa consiste en obtener las avenidas de diseño para diversos períodos de retorno y simular su tránsito por el vaso de acuerdo con las reglas de operación de la obra de excedencias, suponiendo que dichas avenidas se presentan cuando el nivel en el vaso se encuentra en el nivel de aguas máximas de operación (NAMO).

En este trabajo se presenta una metodología para evaluar el riesgo de inundaciones aguas abajo de una presa tomando en cuenta que, cuando se presentan las avenidas de diseño, el nivel en el vaso es una variable aleatoria que depende de la política de operación a largo plazo de la presa. Para la aplicación de la metodología propuesta se utiliza el caso de la presa El Cuchillo, situada en el estado de Nuevo León en México; se presentan primero los métodos usados para la estimación de las avenidas asociadas a distintos períodos de retorno, así como para la definición de la política de operación a largo plazo de la presa, de manera que al simular con esta última el funcionamiento de la presa se deducen las probabilidades asociadas al nivel en el embalse durante la época de avenidas.

Al simular el tránsito de las avenidas asociadas a cada período de retorno se obtienen las probabilidades condicionales asociadas a los caudales máximos de descarga, para finalmente estimar el riesgo de inundaciones aguas abajo de la presa.
\end{abstract}

Palabras clave: Políticas de operación, frecuencia, afectaciones, costos, curva guía, niveles óptimos, riesgo.

\begin{abstract}
The common practice to assess the flooding risk downstream of a dam is to obtain design floods for different return periods and simulate their transit through the reservoir according to the operation rules of the spillway, assuming that those floods occur when the level in the reservoir is in the normal water level $(N W L)$.

This paper presents a methodology for assessing the flooding risk downstream of a dam considering that the level in the reservoir when the design flood is presented, is a random variable that depends on the long-term operation policy of the dam. For the application of the proposed methodology El Cuchillo Dam is used, located in Nuevo Leon state in Mexico, the methods used for estimating floods associated to different return periods and for defining the long-term operation policy of the dam are presented
\end{abstract}

\footnotetext{
1 Programa de Maestría y Doctorado en Ingeniería. Universidad Nacional Autónoma de México. Cd. Universitária. CP. 04510. México, DF. E-mail: hulosafa@hotmail.com

2 Instituto de Ingeniería. Universidad Nacional Autónoma de México. Cd. Universitária. CP. 04510. México, DF. E-mail: RDominguezM@iingen.unam.mx

* Autor de correspondencia
} 
first; therefore, simulating the dam operation based in that policy, the probabilities associated with the reservoir level during flood period are deducted.

When simulating flood routing associated to each return period, conditional probabilities associated with the maximum discharge flow are obtained, and finally the flooding risk downstream of the dam can be estimated.

Keywords: Operating policies, frequency, affectations, cost, guide curve, optimum levels, risk.

\section{INTRODUCCIÓN}

El problema de determinar la política de operación óptima a largo plazo en un sistema de presas ha sido el tema de numerosas investigaciones y publicaciones en los últimos años.

Rebolledo S. [1] analiza la "Operación óptima de un sistema hidráulico formado por dos presas en paralelo"; Avilés H.R. [2] la "Optimación en línea de presas hidroeléctricas"; Contreras C.C. [3] la "Operación óptima de sistema de presas en cascada"; Arganis J.M. [4] estudia la "Operación óptima de un sistema de presas en cascada para generación hidroeléctrica tomando en cuenta condiciones reales de operación y el uso de muestras sintéticas para el pronóstico".

Sánchez C. y Wagner G., [5] presentan la "Determinación de reglas de operación óptima para dos embalses, utilizando un algoritmo genético" y en el 2004 el "Modelo numérico para la operación óptima de un hidrosistema de aguas superficiales" [6].

En particular la operación óptima del sistema de presas del río Grijalva ha sido objeto de estudio. Al respecto, Domínguez M.R. [7] plantea el problema de determinar políticas de operación mensual que maximizan una función objetivo que toma en cuenta la generación de energía a largo plazo y que procura evitar derrames y déficit; Domínguez M.R. [8] complementa el estudio de 1993, definen políticas de extracción trabajando el sistema de presas. La Angostura y Malpaso, en función del almacenamiento final del mes previo en ambas presas; Domínguez M.R. [9] hace una revisión del funcionamiento y operación de las presas del río Grijalva; Domínguez M.R. [10] adapta el modelo de optimación de políticas de extracciones del sistema de presas, considerando el valor relativo de la energía "de pico" respecto a la "de base", incorporando restricciones de energía mínima propuesta por la Comisión Federal de
Electricidad (CFE); Domínguez M.R. [11] genera nuevas políticas de operación, que toman en cuenta los eventos hidrológicos ocurridos en el 2005.

Por otra parte, Dittmann, Froehlich, Pohl y Ostrowski [12], proponen un funcionamiento dinámico a largo plazo mediante el uso de algoritmos evolutivos, demostrando que el estado de funcionamiento dinámico es superior a la regla de operación estática.

Vigyan B.J. [13] realiza el planteamiento de tres curvas guía de operación para la presa de Tawa en India, las que se ajustan a las diversas demandas y propósitos del embalse.

Pradhan S. N., Tripathy U.K. [14] optimizan la operación del embalse multipropósito de Hirakud (India), empleando un algoritmo genético reflejando la eficiencia de los resultados.

Sin embargo, en estos trabajos no se considera explícitamente el efecto que producen las políticas obtenidas en el riesgo de inundaciones aguas abajo de las presas, por lo que este trabajo se enfoca al análisis de las políticas de operación en los embalses considerando el riesgo asociado a las afectaciones aguas abajo de las presas.

\section{SITIO DE ESTUDIO}

El río Bravo tiene una longitud de 3.034 km y drena una área de $607.965 \mathrm{~km}^{2}$.

Nace en las montañas San Juan en el Estado de Colorado, EE.UU., fluye por medio del valle de San Luis hacia el sur, pasando por Nuevo México por medio de Albuquerque y Las Cruces hacia El Paso, Texas (Figura 1).

Actualmente el río Bravo es sobrexplotado, es decir, la demanda de agua excede la capacidad de abastecimiento del río. 


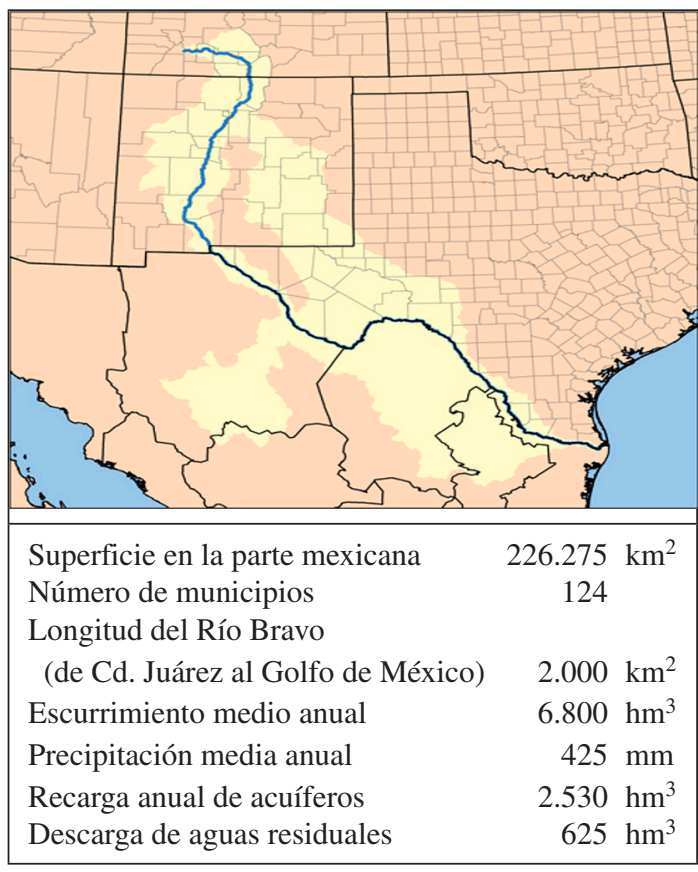

Figura 1. Características generales de la cuenca del río Bravo.

En la cuenca del Río Bravo/Grande se han construido un gran número de presas como La Amistad, Caballo, El Cuchillo, Elephant Butte, F. Madero, Falcón, Luis L. León, Red Bluff y San Gabriel, La Boca, Cerro Prieto, Marte R. Gómez, Las Blancas, entre otras; de las que, la presa El Cuchillo, ubicada dentro de la Subcuenca del río San Juan, se tomará como ejemplo para desarrollar la presente investigación (Figura 2).

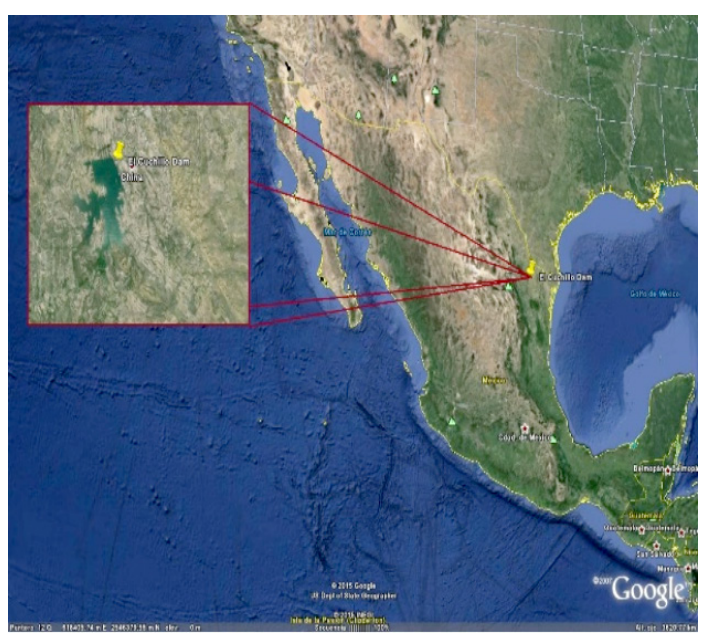

Figura 2. Ubicación de la presa El Cuchillo.

\section{METODOLOGÍA}

Existen diversos criterios para determinar las avenidas que se emplean en el diseño de una obra de excedencias; sin embargo, cualquier metodología que se use para tal fin debe cumplir tres aspectos:

i. Que considere la correlación pico-volumen,

ii. Que permita reproducir avenidas similares a las observadas en el período de registro, y

iii. Que conduzca a la definición de distintos tipos de avenidas que, en cada caso, podrán modificar los resultados según las características del vaso en donde se transiten.

La metodología propuesta consiste de los siguientes pasos:

a) Análisis de los registros

A fin de garantizar la confiabilidad de los registros (ya sean horarios, diarios o mensuales) se analiza la congruencia de los mismos, revisando que no se presenten errores de captura, los que puedan afectar significativamente el desarrollo del análisis.

b) Avenidas de diseño

El procedimiento utilizado permite estimar la forma de la avenida de diseño a partir del análisis de los caudales medios diarios históricamente registrados. Para ello se determinan los caudales medios máximos anuales para distintas duraciones. El caudal medio máximo para una duración de un día corresponde al caudal medio diario máximo anual. Para obtener los caudales medios máximos para las otras duraciones se procede a encontrar para cada año de registro el promedio máximo para $n$ días consecutivos según la duración que se analice:

$$
\bar{Q}_{M_{n}}=\max _{i}\left(\frac{\sum_{i}^{i+n-1} Q_{k}}{n}\right)
$$

donde

$\bar{Q}_{M_{n}}$ caudal medio máximo para $n$ días de duración $Q_{k} \quad$ caudal medio diario el día $k$

$n \quad$ duración en días contador del día en que inicia el lapso de duración $n$. 
De esta forma, para cada valor de duración (1, $2, \ldots, n)$ se cuenta con una muestra de $m$ valores de caudales máximos anuales, a los que se puede ajustar una función de distribución. Las funciones de distribución ajustadas (una para cada duración) permiten entonces estimar la avenida (sintética) de diseño para cualquier período de retorno $\left(T_{r}\right)$.

La construcción de curvas del tipo Caudal -DuraciónPeriodo de retorno de los eventos extrapolados permite observar su comportamiento, tomando en cuenta que la tendencia que se obtiene para períodos de retorno menores o iguales que el número de años de registro debe conservarse para períodos de retorno mayores, de manera que si esto no ocurre, deben revisarse las extrapolaciones.

Corroborando lo anterior, es necesario pasar de las avenidas sintéticas a avenidas reales. Para ello, con cada período de retorno que se seleccione, primero se convierten los caudales medios asociados a distintas duraciones en caudales medios diarios mediante las ecuaciones recursivas:

$$
\begin{gathered}
q_{1}\left(T_{r}\right)=\bar{Q}_{1}\left(T_{r}\right) \\
q_{k}\left(T_{r}\right)=k \bar{Q}_{k}\left(T_{r}\right)-(k-1) \bar{Q}_{k-1}\left(T_{r}\right)
\end{gathered}
$$

donde

$$
\begin{aligned}
& \bar{Q}_{k}\left(T_{r}\right) \begin{array}{l}
\text { caudal medio estimado para una duración } \\
\text { de } k \text { días y un período de retorno } T_{r}
\end{array} \\
& q_{k}\left(T_{r}\right) \quad \begin{array}{l}
\text { caudal medio diario, } \mathrm{m}^{3} / \mathrm{s} .
\end{array}
\end{aligned}
$$

Finalmente, los caudales medios diarios $q_{k}\left(T_{r}\right)$, cuya secuencia tiene una tendencia decreciente, deben de reordenarse para que tomen la forma de un hidrograma histórico representativo, una forma sencilla de hacer el ordenamiento es el uso del método de bloques alternos, en el que al centro de la avenida se coloca el caudal individual de un día; hacia adelante se coloca el caudal de dos días, hacia atrás del caudal del centro se coloca el de tres días y así, se van colocando el de cuatro días hacia adelante, el de cinco días hacia atrás, hasta construir la forma de la avenida.

\section{c) Operación óptima}

Con la finalidad de mejorar la operación de la presa en estudio, se optimiza la misma, asignando diversos tipos de restricciones y penalizaciones, ya sea por derrame o déficit de agua; para ello, se integran los registros en intervalos quincenales con la finalidad de realizar su análisis en políticas a largo plazo. Lo anterior, a manera de repartir el volumen en intervalos semejantes durante todo el año considerando la época de avenidas y de estiaje. Partiendo del volumen útil de la presa, se define el número de estados que contendrá cada uno de los grupos conformados.

Con dichos grupos, mediante el empleo de la programación dinámica estocástica, se determinan los máximos beneficios esperados en un horizonte de planeación de $N$ etapas que lleven a la definición de políticas de operación óptima, para todos los posibles estados iniciales y extracciones de una presa.

Para llevar a cabo la optimización se emplea como función objetivo la maximización del valor esperado del beneficio total por generación, imponiendo penalizaciones por déficit o derrames en la presa.

$$
F_{O b j}=\operatorname{Max} E\left(G E-C_{1} D E R R-C_{2} D E F\right)
$$

donde

$E(\quad)$ operador valor esperado

$G E$ energía generada, GWh

DERR derrame, $\mathrm{hm}^{3}$

$D E F$ déficit, $\mathrm{hm}^{3}$

$C_{1} \quad$ coeficiente de penalización por derrame $C_{2} \quad$ coeficiente de penalización por déficit.

Partiendo de dicha optimización, se efectúa la simulación analítica del vaso, comparando los niveles obtenidos con respecto a los históricos.

\section{d) Tránsito de avenidas}

Como resultado del funcionamiento de vaso optimizado se obtienen los niveles más frecuentes en el embalse (histograma de elevaciones medias), los que serán las condiciones de inicio en el tránsito de las avenidas de diseño generando un escenario de análisis que parte de niveles asociados a su probabilidad de ocurrencia $\left(P_{\text {Elev }}\right)$.

Se efectúa el tránsito de las avenidas estimadas para $2,5,10,20,50,100,200,500,1.000,2.000,5.000$ y 10.000 años de periodo de retorno y para cada elevación inicial obtenida de la operación óptima, 
asimismo se analiza otro escenario considerando como elevación inicial la del NAMO del embalse.

Del resultado de la simulación se obtienen los caudales máximos de descarga, los que son asociados a su probabilidad de excedencia $\left(P_{Q}\right)$. Con la finalidad de determinar caudales máximos de descarga en cifras cerradas $(1.000,2.000$, etc.) se grafican los resultados obtenidos determinando la tendencia a la cual se ajustan (Figura 3), para asociarles su probabilidad de excedencia y periodo de retorno correspondientes (Tabla 1).

e) Análisis de la probabilidad de excedencia conjunta

Para determinar la probabilidad de excedencia conjunta asociada al caudal máximo de descarga se realiza la suma total de los productos de cada probabilidad de ocurrencia asociada a la elevación inicial $\left(P_{\text {Elev }}\right)$ por la probabilidad de excedencia $\left(P_{Q}\right)$, empleando la fórmula:

$$
P_{T i}=\sum_{k=1}^{n} P_{Q_{i}} P_{\text {Elev }_{k}}
$$

donde

$P_{T i} \quad$ probabilidad de excedencia conjunta para un caudal $Q_{i}$

$P_{Q i} \quad$ probabilidad de excedencia del caudal $Q_{i}$ dada una elevación inicial $k$

\section{Elevación inicial 156 MSNM}

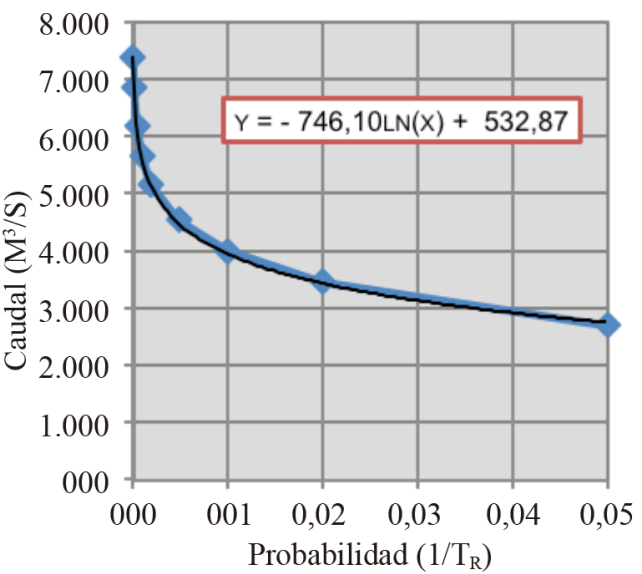

Figura 3. Curva caudal máximo de descarga vs. Probabilidad de excedencia.
$P_{\text {Elev }}$ probabilidad de ocurrencia asociada a la elevación inicial $k$.

Tabla 1. Probabilidad de excedencia conjunta.

\begin{tabular}{|c|c|c|c|c|}
\hline Elev Inicial & \multicolumn{2}{|c|}{ Caudal $\left(\mathrm{m}^{3} / \mathrm{s}\right)$} & \multicolumn{2}{|c|}{8,000} \\
\hline msnm & $T_{r}$ & $P_{Q}$ & $\boldsymbol{P}_{\text {Elev }}$ & $\boldsymbol{P}_{Q} \boldsymbol{P}_{\text {Elev }}$ \\
\hline 156 & $22,208.18$ & 0,00005 & 0,11538 & 0,000005 \\
\hline 158 & $14,155.66$ & 0,00007 & 0,18269 & 0,000013 \\
\hline 160 & $6,251.65$ & 0,00016 & 0,34615 & 0,000055 \\
\hline 162 & $3,526.60$ & 0,00028 & 0,35577 & 0,000101 \\
\hline & & & $P_{T} 8.000$ & 0,000174 \\
\hline
\end{tabular}

En la Tabla 1 se ejemplifican los cálculos correspondientes a un caudal de $8.000 \mathrm{~m}^{3} / \mathrm{s}$.

Como resultado se obtendrán tantas probabilidades de excedencia conjunta $\left(P_{T}\right)$ como caudales hayan sido analizados, los que deberán ser comparados con los resultados obtenidos considerando como elevación inicial el NAMO.

f) Tránsito hidráulico por el cauce y costos por afectación

Empleando los caudales máximos de descarga seleccionados se realiza el tránsito hidráulico por el cauce, aguas abajo del embalse, determinando las áreas afectadas por la inundación.

Dicha área es multiplicada por un costo unitario de afectación, determinando los costos asociados a diversas probabilidades de excedencia.

Finalmente, al generar la curva Costos vs. Probabilidades de excedencia conjunta, se determina el riesgo esperado, definido por el área bajo la curva, el que puede compararse con el obtenido considerando el NAMO como elevación inicial.

\section{APLICACIÓN}

\section{Análisis de los caudales medios mensuales}

Con la finalidad de validar las avenidas de diseño de la presa El Cuchillo, se llevó acabo su actualización partiendo de los registros diarios proporcionados por la Comisión Nacional del Agua (CONAGUA). 
Para tal efecto, se calcularon las aportaciones al vaso de almacenamiento considerando los ingresos por cuenca propia como la suma del incremento (o decremento) en el almacenamiento (DELTA V) + las salidas medidas (SALIDAS). De lo anterior, se obtuvo una muestra de caudales medios diarios para un periodo de registro del año 1995 a 2009 (Tabla 2),

Tabla 2. Caudales medios mensuales Presa El Cuchillo, $\mathrm{m}^{3} / \mathrm{s}$.

\begin{tabular}{|l|r|r|r|r|r|r|}
\hline \multicolumn{1}{|c|}{ Año } & Ene & Feb & Mar & Abr & May & Jun \\
\hline 1995 & 1,60 & 0,51 & 0,21 & 0,10 & 1,60 & 1,64 \\
\hline 1998 & 1,51 & 0,60 & 0,80 & 0,13 & 0,01 & 1,05 \\
\hline 1999 & 1,99 & 0,28 & 0,65 & 1,29 & 1,86 & 9,16 \\
\hline 2000 & 0,94 & 0,68 & 0,19 & 0,47 & 1,87 & 3,44 \\
\hline 2001 & 3,15 & 2,23 & 0,97 & 0,69 & 3,65 & 2,74 \\
\hline 2002 & 6,42 & 2,30 & 0,96 & 0,98 & 0,37 & 1,19 \\
\hline 2003 & 23,82 & 15,21 & 10,46 & 3,89 & 8,25 & 17,24 \\
\hline 2004 & 18,80 & 11,45 & 29,60 & 65,07 & 40,21 & 12,82 \\
\hline 2005 & 7,86 & 11,04 & 14,38 & 4,87 & 14,33 & 2,39 \\
\hline 2006 & 15,42 & 8,57 & 5,18 & 2,86 & 6,36 & 3,60 \\
\hline 2007 & 10,86 & 10,75 & 3,77 & 6,38 & 13,30 & 12,20 \\
\hline 2008 & 5,96 & 2,45 & 0,71 & 1,18 & 1,41 & 0,47 \\
\hline 2009 & 10,30 & 6,03 & 3,13 & 0,89 & 3,69 & 1,05 \\
\hline Promedio & 8,36 & 5,55 & 5,46 & 6,83 & 7,45 & 5,31 \\
\hline Max. & 23,82 & 15,21 & 29,60 & 65,07 & 40,21 & 17,24 \\
\hline Min. & 0,94 & 0,28 & 0,19 & 0,10 & 0,01 & 0,47 \\
\hline Mediana & 6,42 & 2,45 & 0,97 & 1,18 & 3,65 & 2,74 \\
\hline Des. est. & 7,26 & 5,22 & 8,46 & 17,61 & 10,89 & 5,57 \\
\hline Coef. var. & 0,87 & 0,94 & 1,55 & 2,58 & 1,46 & 1,05 \\
\hline
\end{tabular}

\begin{tabular}{|l|r|r|r|r|r|r|}
\hline \multicolumn{1}{|c|}{ Año } & Jul & Ago & Sep & Oct & Nov & Dic \\
\hline 1995 & 1,25 & 108,54 & 45,93 & 7,59 & 3,61 & 2,22 \\
\hline 1998 & 0,02 & 1,25 & 43,60 & 21,94 & 1,31 & 0,07 \\
\hline 1999 & 15,61 & 14,37 & 8,83 & 9,68 & 1,77 & 1,09 \\
\hline 2000 & 0,34 & 0,14 & 7,64 & 65,75 & 8,72 & 5,27 \\
\hline 2001 & 1,63 & 1,83 & 138,53 & 39,44 & 37,02 & 12,64 \\
\hline 2002 & 13,92 & 1,25 & 128,46 & 54,33 & 92,02 & 22,19 \\
\hline 2003 & 16,42 & 20,53 & 215,27 & 177,86 & 47,26 & 15,00 \\
\hline 2004 & 10,80 & 9,80 & 151,08 & 53,19 & 18,77 & 10,73 \\
\hline 2005 & 105,11 & 39,91 & 38,11 & 122,15 & 40,31 & 17,79 \\
\hline 2006 & 4,93 & 2,91 & 40,64 & 19,77 & 11,76 & 11,99 \\
\hline 2007 & 37,89 & 20,65 & 38,84 & 12,08 & 6,41 & 4,05 \\
\hline 2008 & 10,06 & 18,46 & 409,05 & 71,66 & 29,43 & 15,88 \\
\hline 2009 & 0,60 & 2,99 & 43,96 & 24,43 & 13,48 & 18,12 \\
\hline Promedio & 16,81 & 18,67 & 100,76 & 52,30 & 23,99 & 10,54 \\
\hline Max. & 105,11 & 108,54 & 409,05 & 177,86 & 92,02 & 22,19 \\
\hline Min. & 0,02 & 0,14 & 7,64 & 7,59 & 1,31 & 0,07 \\
\hline Mediana & 10,06 & 9,80 & 43,96 & 39,44 & 13,48 & 11,99 \\
\hline Des. est. & 28,52 & 29,38 & 112,13 & 49,63 & 25,64 & 7,29 \\
\hline Coef. var. & 1,70 & 1,57 & 1,11 & 0,95 & 1,07 & 0,69 \\
\hline
\end{tabular}

sin considerar los años 1996 y 1997 debido a que sus datos son incompletos.

\section{Actualización de las avenidas}

Se realizó un análisis probabilístico para el ajuste de los valores medios diarios máximos anuales, mediante el empleo de funciones de distribución de probabilidad, lo que permitió la obtención de valores extrapolados a diferentes períodos de retorno.

Para realizar los cálculos correspondientes a los ajustes, para las diferentes funciones de distribución de probabilidad, se utilizó el programa AX@ [15].

Los registros medios diarios máximos para duraciones de 1 a 15 días [16] se analizaron estadísticamente con distintas funciones de distribución, seleccionando la función Doble Gumbel [17-18] como la de mejor ajuste. Para cada duración se determinaron los caudales medios máximos asociados a distintos períodos de retorno $(2,5,10,20,50,100,200,500,1.000$, $2.000,5.000$ y 10.000 años) y se revisó su tendencia mediante la construcción de las curvas caudales -duración- período de retorno (Figura 4).

A partir de esos resultados se convirtieron las avenidas sintéticas en reales, determinando la

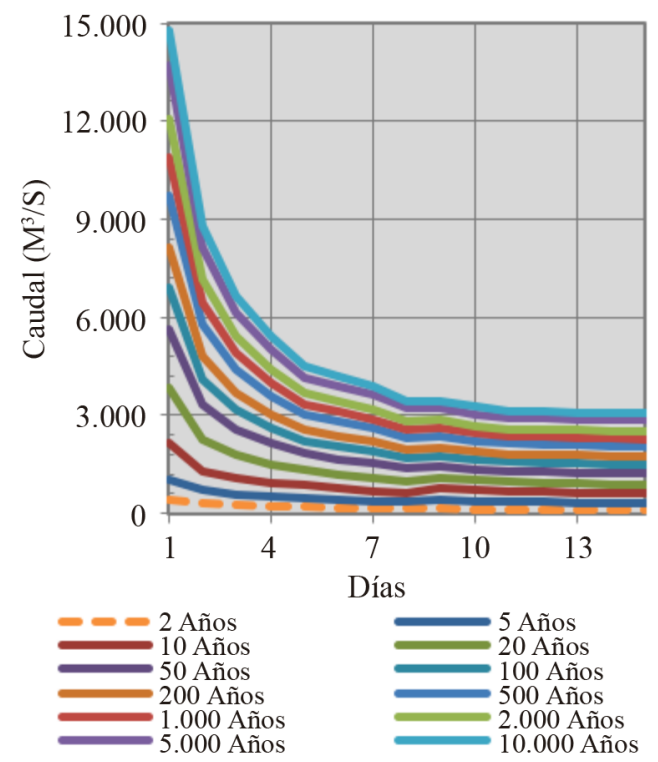

Figura 4. Curva caudales -duración- período de retorno. 
forma de la avenida mediante el método de alternar bloques [19], en el que a la mitad de la duración total se coloca el valor máximo $\left(Q_{1}\right)$, hacia delante se coloca el caudal $\left(Q_{2}\right)$, hacia atrás el caudal $\left(Q_{3}\right)$, y así sucesivamente. Los resultados obtenidos para las avenidas asociadas a diversos períodos de retorno se aprecian en la Tabla 3 y de manera gráfica en la Figura 5.

\section{Operación óptima}

Considerando los caudales medios diarios presentados en la Tabla 2, así como las características

Tabla 3. Caudales medios diarios para distintos eventos de diseño, en $\mathrm{m}^{3} / \mathrm{s}$.

\begin{tabular}{|r|r|r|r|r|r|r|}
\hline $\boldsymbol{T}_{\boldsymbol{r}}$ & $\mathbf{2}$ & \multicolumn{1}{|c|}{$\mathbf{5}$} & \multicolumn{1}{|c|}{$\mathbf{1 0}$} & \multicolumn{1}{|c|}{$\mathbf{2 0}$} & $\mathbf{5 0}$ & $\mathbf{1 0 0}$ \\
\hline 1 & 62 & 105 & 247 & 277 & 294 & 308 \\
\hline 2 & 63 & 126 & 251 & 474 & 600 & 720 \\
\hline 3 & 67 & 132 & 298 & 569 & 803 & 952 \\
\hline 4 & 76 & 154 & 368 & 594 & 934 & 1.176 \\
\hline 5 & 101 & 203 & 408 & 665 & 947 & 1.204 \\
\hline 6 & 117 & 254 & 465 & 706 & 1.095 & 1.321 \\
\hline 7 & 190 & 372 & 682 & 910 & 1.152 & 1.376 \\
\hline 8 & 465 & 1.047 & 2.177 & 3.886 & 5.689 & 6.943 \\
\hline 9 & 203 & 834 & 1.879 & 1.844 & 1.986 & 2.151 \\
\hline 10 & 166 & 342 & 507 & 706 & 1.097 & 1.371 \\
\hline 11 & 106 & 216 & 415 & 676 & 1.009 & 1.250 \\
\hline 12 & 92 & 187 & 369 & 621 & 938 & 1.180 \\
\hline 13 & 72 & 137 & 336 & 590 & 835 & 971 \\
\hline 14 & 66 & 131 & 254 & 546 & 767 & 947 \\
\hline 15 & 63 & 118 & 247 & 409 & 590 & 637 \\
\hline
\end{tabular}

\begin{tabular}{|c|c|c|c|c|c|c|}
\hline$T_{r}$ & 200 & 500 & 1.000 & 2.000 & 5.000 & 10.000 \\
\hline 1 & 318 & 330 & 351 & 379 & 395 & 275 \\
\hline 2 & 848 & 1.014 & 1.121 & 1.247 & 1.413 & 1.536 \\
\hline 3 & 1.096 & 1.287 & 1.440 & 1.585 & 1.775 & 1.917 \\
\hline 4 & 1.409 & 1.702 & 1.858 & 2.009 & 2.223 & 2.382 \\
\hline 5 & 1.457 & 1.715 & 1.952 & 2.178 & 2.531 & 2.764 \\
\hline 6 & 1.487 & 1.790 & 2.045 & 2.327 & 2.665 & 2.916 \\
\hline 7 & 1.647 & 2.010 & 2.270 & 2.540 & 2.806 & 3.250 \\
\hline 8 & 8.162 & 9.748 & 10.949 & 12.122 & 13.736 & 14.836 \\
\hline 9 & 2.338 & 2.589 & 2.788 & 2.986 & 3.249 & 3.444 \\
\hline 10 & 1.646 & 2.002 & 2.269 & 2.538 & 2.782 & 3.046 \\
\hline 11 & 1.483 & 1.790 & 2.009 & 2.266 & 2.566 & 2.830 \\
\hline 12 & 1.415 & 1.713 & 1.941 & 2.170 & 2.525 & 2.611 \\
\hline 13 & 1.174 & 1.441 & 1.636 & 1.814 & 2.071 & 2.275 \\
\hline 14 & 1.053 & 1.194 & \begin{tabular}{|c|}
1.300 \\
\end{tabular} & 1.405 & 1.519 & 1.727 \\
\hline 15 & 672 & 717 & 759 & 795 & 816 & 853 \\
\hline
\end{tabular}

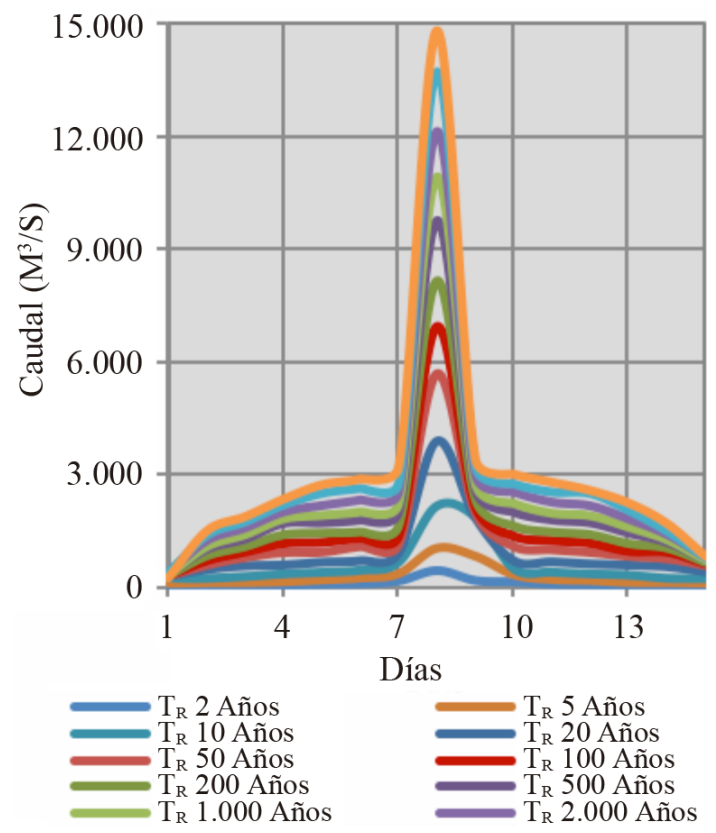

Figura 5. Avenidas de diseño.

del embalse presentadas en la Tabla 4, se realizó la optimización de la operación a largo plazo [2022]. Para lo anterior, la matriz de escurrimientos a emplear en la simulación analítica del vaso se construyó para intervalos quincenales.

Por otra parte, con la finalidad de mitigar las pérdidas posibles por déficit o derrames en la operación de la central, se repartió el volumen medio anual de escurrimientos en intervalos semejantes durante todo el año, es decir, considerando la época de avenidas y estiaje se definieron grupos integrando los meses en el año, con el mes inmediato subsecuente o antecedente, por ejemplo, mayo con abril, o mayo con junio. En la Tabla 5 se presentan los volúmenes y grupos definidos.

Tomando en cuenta el volumen útil de la presa $\left(1.123,00 \mathrm{hm}^{3}\right)$, se determinó repartirlo, en 50 estados compuestos por $22,46 \mathrm{hm}^{3}$ cada uno. Se definieron las frecuencias absolutas de los volúmenes de escurrimiento correspondientes a cada estado y cada grupo de meses definidos en la Tabla 5; las frecuencias relativas, se obtuvieron dividiendo las frecuencias absolutas entre el total de años de la muestra, y se consideraron como una primera aproximación a las probabilidades asociadas a los ingresos. 
Tabla 4. Características generales.

\begin{tabular}{|c|c|c|}
\hline \multicolumn{3}{|l|}{ Generales } \\
\hline Nombre oficial & \multicolumn{2}{|c|}{ Solidaridad } \\
\hline Municipio & \multicolumn{2}{|c|}{ China } \\
\hline Estado & \multicolumn{2}{|c|}{ Nuevo León } \\
\hline Coordenadas & \multicolumn{2}{|c|}{$\begin{array}{c}99^{\circ} 17^{\prime} \text { de } \\
\text { Longitud oeste }\end{array}$} \\
\hline \multicolumn{3}{|l|}{ Hidrología } \\
\hline Área de la cuenca & 8924 & $\mathrm{~km}^{2}$ \\
\hline Escurrimiento mínimo anual & 30,70 & $\mathrm{hm}^{3}$ \\
\hline Escurrimiento medio anual & 666,00 & $\mathrm{hm}^{3}$ \\
\hline Escurrimiento máximo anual & $2.776,50$ & $\mathrm{hm}^{3}$ \\
\hline Caudal de diseño ( $\operatorname{Tr} 10.000$ años) & 15.000 & $\mathrm{~m}^{3} / \mathrm{s}$ \\
\hline Caudal máximo registrado & 7.500 & $\mathrm{~m}^{3} / \mathrm{s}$ \\
\hline \multicolumn{3}{|l|}{ Cortina } \\
\hline Tipo & \multicolumn{2}{|c|}{$\begin{array}{c}\text { Cortina de } \\
\text { materiales graduados }\end{array}$} \\
\hline Elevación de la corona & 169,66 & msnm \\
\hline Longitud de la corona & 4.480 & $\mathrm{~m}$ \\
\hline Altura máxima & 44 & $\mathrm{~m}$ \\
\hline Ancho de la corona & 10 & $\mathrm{~m}$ \\
\hline \multicolumn{3}{|l|}{ Embalse } \\
\hline Azolves & 147,64 & msnm \\
\hline NAMO & 162,35 & msnm \\
\hline NAME & 166,66 & msnm \\
\hline Bordo libre & 3,00 & $\mathrm{~m}$ \\
\hline Capacidad al nivel de Azolves & 100 & $\mathrm{hm}^{3}$ \\
\hline Capacidad al NAMO & 1.123 & $\mathrm{hm}^{3}$ \\
\hline Capacidad NAMO al NAME & 661 & $\mathrm{hm}^{3}$ \\
\hline Capacidad total & 1.784 & $\mathrm{hm}^{3}$ \\
\hline Área al NAMO & 12.795 & ha \\
\hline Área al NAME & 18.092 & ha \\
\hline \multicolumn{3}{|c|}{ Obras de Excedencias } \\
\hline Elevación de la cresta & 151,75 & msnm \\
\hline Longitud total de la cresta & 112 & $\mathrm{~m}$ \\
\hline Caudal máximo observado & 7.500 & $\mathrm{~m}^{3} / \mathrm{s}$ \\
\hline Compuertas & 7 & Radial \\
\hline Dimensiones (ancho $\mathrm{x}$ alto) & $13 \times 16$ & $\mathrm{~m}$ \\
\hline
\end{tabular}

Al graficar las frecuencias relativas contra el intervalo de volúmenes se observaron discontinuidades, por lo que se suavizaron cuidando mantener la forma general y obligando a que la suma fuera igual a 1 . En la Tabla 6 se presentan las frecuencias relativas para cada grupo de meses.
Tabla 5. Matriz de grupos $\left(\mathrm{hm}^{3}\right)$.

\begin{tabular}{|c|r|r|r|}
\hline Año & Grupo 1 & Grupo 2 & Grupo 3 \\
\hline 1995 & 6,32 & 11,88 & 290,72 \\
\hline 1997 & & & 1,30 \\
\hline 1998 & 7,96 & 2,78 & 3,36 \\
\hline 1999 & 7,73 & 70,52 & 38,50 \\
\hline 2000 & 5,93 & 14,85 & 0,37 \\
\hline 2001 & 18,21 & 21,25 & 4,90 \\
\hline 2002 & 27,86 & 41,33 & 3,34 \\
\hline 2003 & 138,69 & 110,76 & 54,99 \\
\hline 2004 & 326,99 & 169,84 & 26,26 \\
\hline 2005 & 98,92 & 326,11 & 106,90 \\
\hline 2006 & 83,31 & 39,57 & 7,81 \\
\hline 2007 & 81,73 & 168,72 & 55,31 \\
\hline 2008 & 27,04 & 31,93 & 49,45 \\
\hline 2009 & 52,86 & 14,23 & 8,02 \\
\hline Media & 65,87 & 78,75 & 46,51 \\
\hline Mediana & 33,26 & 39,57 & 17,14 \\
\hline Desv. est. & 85,55 & 93,80 & 76,72 \\
\hline Coef var. & 0,77 & 0,84 & 0,61 \\
\hline Mínimo & 5,93 & 2,78 & 0,37 \\
\hline Máximo & 326,99 & 326,11 & 290,72 \\
\hline
\end{tabular}

\begin{tabular}{|l|r|r|r|}
\hline Año & Grupo 4 & Grupo 5 & Grupo 6 \\
\hline 1995 & 119,04 & 20,33 & 15,29 \\
\hline 1997 & 3,94 & 7,55 & 22,71 \\
\hline 1998 & 113,00 & 58,75 & 3,58 \\
\hline 1999 & 22,90 & 25,92 & 7,53 \\
\hline 2000 & 19,80 & 176,11 & 36,72 \\
\hline 2001 & 359,06 & 105,62 & 129,81 \\
\hline 2002 & 332,96 & 145,53 & 297,94 \\
\hline 2003 & 557,98 & 476,37 & 162,66 \\
\hline 2004 & 391,59 & 142,47 & 77,39 \\
\hline 2005 & 98,78 & 327,18 & 152,14 \\
\hline 2006 & 105,35 & 52,95 & 62,59 \\
\hline 2007 & 100,68 & 32,36 & 27,47 \\
\hline 2008 & $1.060,26$ & 191,95 & 118,80 \\
\hline 2009 & 113,94 & 65,44 & 83,46 \\
\hline Media & 242,81 & 130,61 & 85,58 \\
\hline Mediana & 113,47 & 85,53 & 69,99 \\
\hline Desv. est. & 287,58 & 132,54 & 81,62 \\
\hline Coef var. & 0,84 & 0,99 & 1,05 \\
\hline Mínimo & 3,94 & 7,55 & 3,58 \\
\hline Máximo & $1.060,26$ & 476,37 & 297,94 \\
\hline
\end{tabular}

\begin{tabular}{|c|l|}
\hline Grupo & \multicolumn{1}{|c|}{ Meses } \\
\hline 1 & Enero + Febrero + Marzo + Abril \\
\hline 2 & Mayo + Junio + Julio \\
\hline 3 & Agosto \\
\hline 4 & Septiembre \\
\hline 5 & Octubre \\
\hline 6 & Noviembre + Diciembre \\
\hline
\end{tabular}


Tabla 6. Frecuencias relativas.

\begin{tabular}{|c|c|c|c|}
\hline Intervalo & Grupo 1 & Grupo 2 & Grupo 3 \\
\hline $0,00-22,46$ & 0,0870 & 0,0855 & 0,2786 \\
\hline $22,47-44,92$ & 0,1304 & 0,2222 & 0,1493 \\
\hline $44,93-67,38$ & 0,2174 & 0,1538 & 0,0995 \\
\hline $67,39-89,84$ & 0,2000 & 0,1026 & 0,0597 \\
\hline $89,85-112,30$ & 0,1000 & 0,0855 & 0,0597 \\
\hline $112,31-134,76$ & 0,0700 & 0,0427 & 0,0597 \\
\hline $134,77-157,22$ & 0,0600 & 0,0342 & 0,0398 \\
\hline $157,23-179,68$ & 0,0500 & 0,0342 & 0,0398 \\
\hline $179,69-202,14$ & 0,0400 & 0,0342 & 0,0398 \\
\hline $202,15-224,60$ & 0,0300 & 0,0256 & 0,0398 \\
\hline $224,61-247,06$ & 0,0200 & 0,0256 & 0,0199 \\
\hline $247,07-269,52$ & & 0,0256 & 0,0199 \\
\hline $269,53-291,98$ & & 0,0256 & 0,0199 \\
\hline $291,99-314,44$ & & 0,0171 & 0,0199 \\
\hline $314,45-336,90$ & & 0,0171 & 0,0100 \\
\hline $336,91-359,36$ & & 0,0171 & 0,0100 \\
\hline $359,37-381,82$ & & 0,0171 & 0,0100 \\
\hline $381,83-404,28$ & & 0,0085 & 0,0100 \\
\hline $404,29-426,74$ & & 0,0085 & 0,0050 \\
\hline $426,75-449,20$ & & 0,0085 & 0,0050 \\
\hline $449,21-471,66$ & & 0,0085 & 0,0050 \\
\hline $471,67-494,12$ & & & \\
\hline Suma & 1,00 & 1,00 & 1,00 \\
\hline Mínimo & 0,0200 & 0,0085 & 0,0050 \\
\hline Media & 0,0913 & 0,0476 & 0,0476 \\
\hline Máximo & 0,2174 & 0,2222 & 0,2786 \\
\hline Intervalo & Grupo 4 & Grupo 5 & Grupo 6 \\
\hline $0,00-22,46$ & 0,0379 & 0,0199 & 0,0495 \\
\hline $22,47-44,92$ & 0,1136 & 0,0397 & 0,1386 \\
\hline $44,93-67,38$ & 0,0758 & 0,1787 & 0,1188 \\
\hline $67,39-89,84$ & 0,0500 & 0,1390 & 0,0891 \\
\hline $89,85-112,30$ & 0,0379 & 0,1191 & 0,0891 \\
\hline $112,31-134,76$ & 0,0417 & 0,0993 & 0,0594 \\
\hline $134,77-157,22$ & 0,0417 & 0,0993 & 0,0594 \\
\hline $157,23-179,68$ & 0,0500 & 0,0596 & 0,0495 \\
\hline $179,69-202,14$ & 0,0583 & 0,0397 & 0,0495 \\
\hline $202,15-224,60$ & 0,0758 & 0,0397 & 0,0495 \\
\hline $224,61-247,06$ & 0,0583 & 0,0397 & 0,0495 \\
\hline $247,07-269,52$ & 0,0500 & 0,0397 & 0,0495 \\
\hline $269,53-291,98$ & 0,0379 & 0,0199 & 0,0495 \\
\hline $291,99-314,44$ & 0,0333 & 0,0199 & 0,0248 \\
\hline $314,45-336,90$ & 0,0250 & 0,0099 & 0,0248 \\
\hline $336,91-359,36$ & 0,0250 & 0,0099 & 0,0248 \\
\hline $359,37-381,82$ & 0,0167 & 0,0050 & 0,0248 \\
\hline $381,83-404,28$ & 0,0167 & 0,0050 & \\
\hline $404,29-426,74$ & 0,0167 & 0,0050 & \\
\hline $426,75-449,20$ & 0,0167 & 0,0050 & \\
\hline & & & \\
\hline
\end{tabular}

\begin{tabular}{|c|c|c|c|}
\hline Intervalo & Grupo 4 & Grupo 5 & Grupo 6 \\
\hline $449,21-471,66$ & 0,0083 & 0,0025 & \\
\hline $471,67-494,12$ & 0,0083 & 0,0025 & \\
\hline $494,13-516,58$ & 0,0083 & 0,0025 & \\
\hline $516,59-539,04$ & 0,0083 & & \\
\hline $539,05-561,50$ & 0,0083 & & \\
\hline $561,51-583,96$ & 0,0083 & & \\
\hline $583,97-606,42$ & 0,0083 & & \\
\hline $606,43-628,88$ & 0,0083 & & \\
\hline $628,89-651,34$ & 0,0083 & & \\
\hline $651,35-673,80$ & 0,0083 & & \\
\hline $673,81-696,26$ & 0,0042 & & \\
\hline $696,27-718,72$ & 0,0042 & & \\
\hline $718,73-741,18$ & 0,0042 & & \\
\hline $741,19-763,64$ & 0,0042 & & \\
\hline $763,65-786,10$ & 0,0042 & & \\
\hline $786,11-808,56$ & 0,0042 & & \\
\hline $808,57-831,02$ & 0,0042 & & \\
\hline $831,03-853,48$ & 0,0042 & & \\
\hline $853,49-875,94$ & 0,0042 & & \\
\hline $875,95-898,40$ & 0,0042 & & \\
\hline Suma & 1,00 & 1,00 & 1,00 \\
\hline Mínimo & 0,0042 & 0,0025 & 0,0248 \\
\hline Media & 0,0251 & 0,0435 & 0,0588 \\
\hline Máximo & 0,1136 & 0,1787 & 0,1386 \\
\hline
\end{tabular}

Con los resultados obtenidos de las frecuencias relativas suavizadas se desarrolló la simulación de la operación óptima del embalse, estimada mediante la programación dinámica estocástica [23].

El resumen anual de los resultados de la simulación de la política de operación óptima se presenta en la Tabla 7. Por otra parte, los promedios quincenales de las variables más relevantes se presentan en la Tabla 8; en particular, los niveles medios quincenales en el embalse se comparan con los históricos como se muestra en la Figura 6. En dicha figura se puede apreciar que la política de operación obtenida de la simulación, conserva los niveles por arriba del régimen medio histórico (con lo que se incrementa la generación), sin llegar al nivel máximo de operación (NAMO).

Partiendo de los niveles en el embalse obtenidos en la simulación, se elaboró el histograma de frecuencias relativas de las elevaciones para los meses de agosto a noviembre (considerándolos como el período de avenidas). Como resultado se conformó la Figura 7 , la cual presenta dichas frecuencias relativas para intervalos de clase de $2 \mathrm{~m}$. 


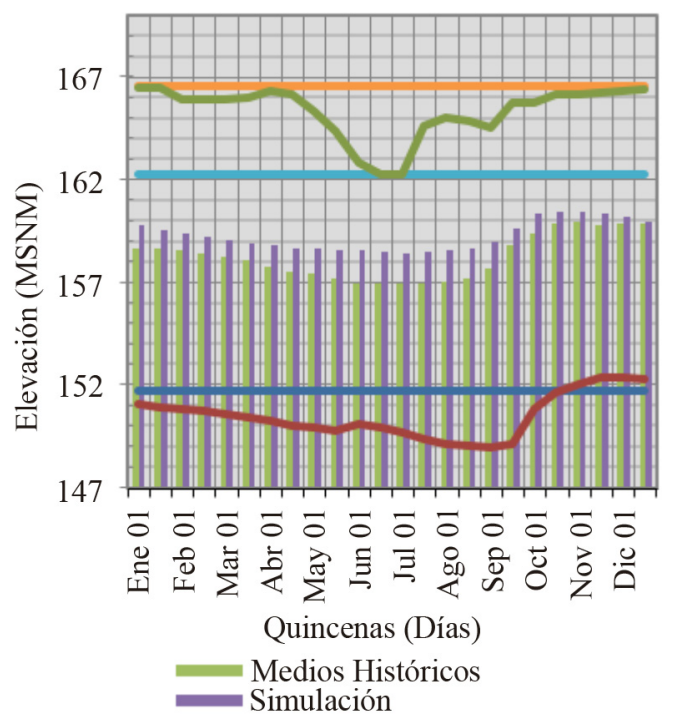

Figura 6. Niveles históricos vs. Operación óptima.

Tabla 7. Funcionamiento analítico del embalse para la política de operación óptima. Resumen anual.

\begin{tabular}{|c|r|c|r|r|}
\hline \multirow{2}{*}{ Año } & Derrame & Déficit & $\begin{array}{c}\text { Ingreso } \\
\text { histórico }\end{array}$ & $\begin{array}{c}\text { Extracción } \\
\text { total }\end{array}$ \\
\cline { 2 - 5 } & \multicolumn{1}{c|}{$\mathbf{h m}^{\mathbf{3}}$} & $\mathbf{h m}^{\mathbf{3}}$ & \multicolumn{1}{c|}{$\mathbf{h m}^{\mathbf{3}}$} & $\mathbf{h m}^{\mathbf{3}}$ \\
\hline 1995 & 0,00 & 0,00 & 463,83 & 626,10 \\
\hline 1998 & 0,00 & 0,00 & 375,59 & 526,50 \\
\hline 1999 & 0,00 & 0,00 & 146,99 & 467,60 \\
\hline 2000 & 0,00 & 0,00 & 453,27 & 359,10 \\
\hline 2001 & 0,00 & 0,00 & 638,48 & 447,70 \\
\hline 2002 & 0,00 & 0,00 & 823,72 & 698,70 \\
\hline 2003 & 416,74 & 0,00 & $1.490,81$ & $1.267,10$ \\
\hline 2004 & 82,27 & 0,00 & $1.139,78$ & $1.139,80$ \\
\hline 2005 & 287,74 & 0,00 & $1.112,78$ & $1.133,60$ \\
\hline 2006 & 0,00 & 0,00 & 349,26 & 688,30 \\
\hline 2007 & 0,00 & 0,00 & 450,84 & 508,60 \\
\hline 2008 & 493,59 & 0,00 & $1.458,05$ & $1.040,40$ \\
\hline 2009 & 0,00 & 0,00 & 338,47 & 718,40 \\
\hline Promedio & 98,49 & 0,00 & 710,91 & 740,15 \\
\hline Suma & $1.280,34$ & 0,00 & $9.241,87$ & $9.621,90$ \\
\hline & & & & \\
\hline
\end{tabular}

\begin{tabular}{|c|c|c|c|}
\hline \multirow{2}{*}{ Año } & $\begin{array}{c}\text { Almacen. } \\
\text { promedio }\end{array}$ & $\begin{array}{c}\text { Elevación } \\
\text { promedio }\end{array}$ & $\begin{array}{c}\text { Energía } \\
\text { total }\end{array}$ \\
\cline { 2 - 4 } & $\mathbf{h m}^{\mathbf{3}}$ & $\mathbf{m s n m}$ & $\mathbf{G W h}$ \\
\hline 1995 & 849,66 & 160,04 & 28,93 \\
\hline 1998 & 754,93 & 159,08 & 23,03 \\
\hline 1999 & 571,47 & 157,05 & 18,22 \\
\hline 2000 & 525,51 & 156,49 & 13,02 \\
\hline 2001 & 578,32 & 157,14 & 17,09 \\
\hline
\end{tabular}

\begin{tabular}{|c|c|c|c|}
\hline \multirow{2}{*}{ Año } & $\begin{array}{c}\text { Almacen. } \\
\text { promedio }\end{array}$ & $\begin{array}{c}\text { Elevación } \\
\text { promedio }\end{array}$ & $\begin{array}{c}\text { Energía } \\
\text { total }\end{array}$ \\
\cline { 2 - 4 } & $\mathbf{h m}^{\mathbf{3}}$ & $\mathbf{m s n m}$ & $\mathbf{G W h}$ \\
\hline 2002 & 801,11 & 159,55 & 31,00 \\
\hline 2003 & 972,36 & 161,10 & 40,76 \\
\hline 2004 & $1.015,17$ & 161,47 & 51,16 \\
\hline 2005 & 989,32 & 161,25 & 41,09 \\
\hline 2006 & 877,42 & 160,28 & 31,77 \\
\hline 2007 & 635,92 & 157,85 & 20,46 \\
\hline 2008 & 732,72 & 158,86 & 23,30 \\
\hline 2009 & 878,94 & 160,30 & 33,32 \\
\hline Promedio & 783,30 & 159,27 & 28,71 \\
\hline Suma & - & - & 373,15 \\
\hline
\end{tabular}

Tabla 8. Funcionamiento analítico del embalse para la política de operación óptima. Resumen quincenal.

\begin{tabular}{|c|c|c|c|c|}
\hline \multirow{2}{*}{ Quincena } & \multirow{2}{*}{ Mes } & $\begin{array}{l}\text { Elevación } \\
\text { promedio }\end{array}$ & $\begin{array}{l}\text { Almacen. } \\
\text { promedio }\end{array}$ & $\begin{array}{c}\text { Energía } \\
\text { total }\end{array}$ \\
\hline & & msnm & $\mathrm{hm}^{3}$ & GWh \\
\hline $1 \mathrm{Q}$ & \multirow{2}{*}{ ENE } & 159,75 & 826,45 & 1,43 \\
\hline $2 Q$ & & 159,57 & 805,92 & 1,39 \\
\hline $1 \mathrm{Q}$ & \multirow{2}{*}{ FEB } & 159,38 & 785,95 & 1,26 \\
\hline $2 \mathrm{Q}$ & & 159,19 & 765,27 & 1,17 \\
\hline $1 \mathrm{Q}$ & \multirow{2}{*}{ MAR } & 159,01 & 749,38 & 1,00 \\
\hline $2 Q$ & & 158,88 & 736,89 & 0,90 \\
\hline $1 \mathrm{Q}$ & \multirow{2}{*}{$\mathrm{ABR}$} & 158,76 & 726,76 & 0,86 \\
\hline $2 \mathrm{Q}$ & & 158,66 & 717,96 & 0,74 \\
\hline $1 \mathrm{Q}$ & \multirow{2}{*}{ MAY } & 158,60 & 716,14 & 0,57 \\
\hline $2 \mathrm{Q}$ & & 158,57 & 712,21 & 0,56 \\
\hline $1 \mathrm{Q}$ & \multirow{2}{*}{ JUN } & 158,52 & 705,86 & 0,53 \\
\hline $2 \mathrm{Q}$ & & 158,47 & 702,30 & 0,53 \\
\hline $1 \mathrm{Q}$ & \multirow{2}{*}{ JUL } & 158,42 & 696,25 & 0,51 \\
\hline $2 \mathrm{Q}$ & & 158,48 & 717,66 & 0,48 \\
\hline $1 \mathrm{Q}$ & \multirow{2}{*}{ AGO } & 158,57 & 717,75 & 1,05 \\
\hline $2 \mathrm{Q}$ & & 158,62 & 730,67 & 1,09 \\
\hline $1 \mathrm{Q}$ & \multirow{2}{*}{ SEP } & 158,98 & 789,78 & 1,80 \\
\hline $2 \mathrm{Q}$ & & 159,62 & 861,52 & 1,95 \\
\hline $1 \mathrm{Q}$ & \multirow{2}{*}{ OCT } & 160,33 & 910,30 & 2,09 \\
\hline $2 \mathrm{Q}$ & & 160,43 & 906,96 & 2,12 \\
\hline $1 \mathrm{Q}$ & \multirow{2}{*}{ NOV } & 160,43 & 908,92 & 1,49 \\
\hline $2 Q$ & & 160,38 & 893,89 & 1,67 \\
\hline $1 \mathrm{Q}$ & \multirow{2}{*}{ DIC } & 160,19 & 869,65 & 1,77 \\
\hline $2 \mathrm{Q}$ & & 159,96 & 844,64 & 1,75 \\
\hline \multicolumn{2}{|l|}{ Suma } & - & - & 28,71 \\
\hline \multicolumn{2}{|c|}{ Promedio } & 159,22 & 781,42 & - \\
\hline
\end{tabular}




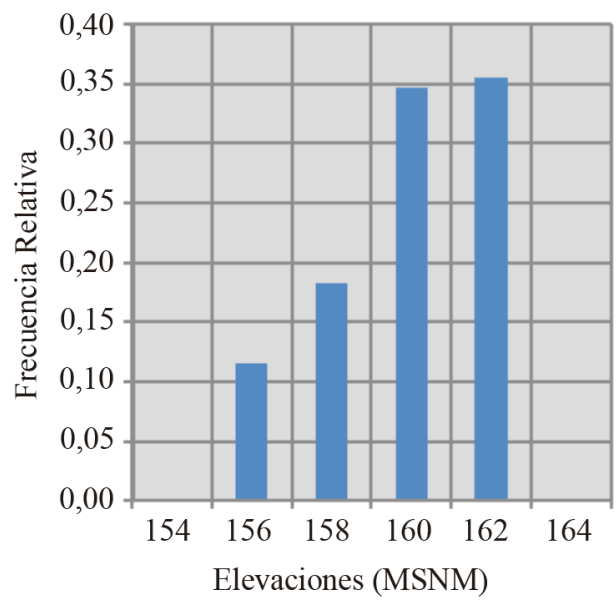

Figura 7. Histograma de elevaciones medias más frecuentes.

\section{Tránsito de avenidas}

Se llevó a cabo la simulación del tránsito de avenidas considerando diferentes niveles iniciales en el embalse, con la finalidad de obtener la magnitud del caudal máximo de descarga correspondiente.

Para la simulación del tránsito de las avenidas, se empleó la curva Elevaciones - Capacidades mostrada en la Figura 8.

Por otra parte, en la Figura 9 se muestra la curva Elevaciones - Caudales del vertedor.

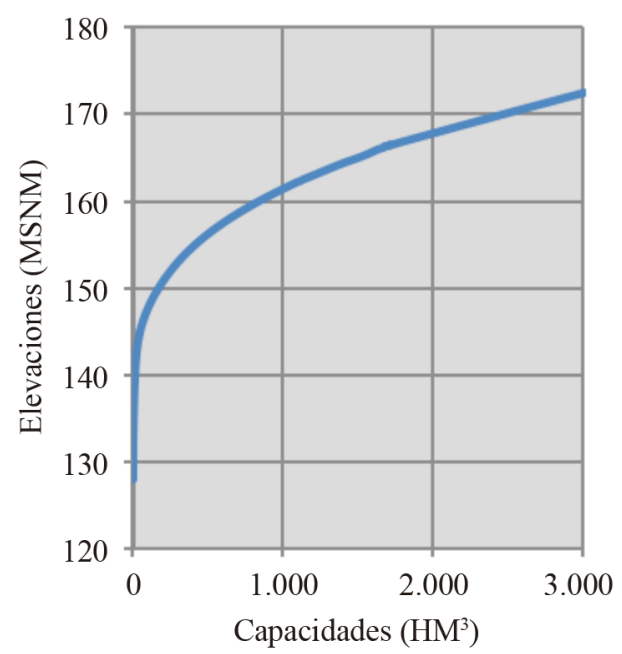

Figura 8. Curva Elevaciones - Capacidades.
Para el análisis de los tránsitos de avenidas se realizó la simulación, mediante el empleo del programa "Tránsito de una avenida por un vaso" [24]. Para tal efecto se consideraron dos escenarios posibles:

1. Partiendo como elevación inicial de la cota del NAMO (162,35 msnm).

2. Tomando como elevaciones iniciales las correspondientes a los cuatro niveles más frecuentes obtenidos de la simulación analítica del embalse (Figura 7).

Las avenidas de entrada al vaso son las presentadas en la Figura 5 y la Tabla 3, las cuales corresponden a los períodos de retorno de 2, 5, 10, 20, 50, 100, $200,500,1.000,2.000,5.000$ y 10.000 años.

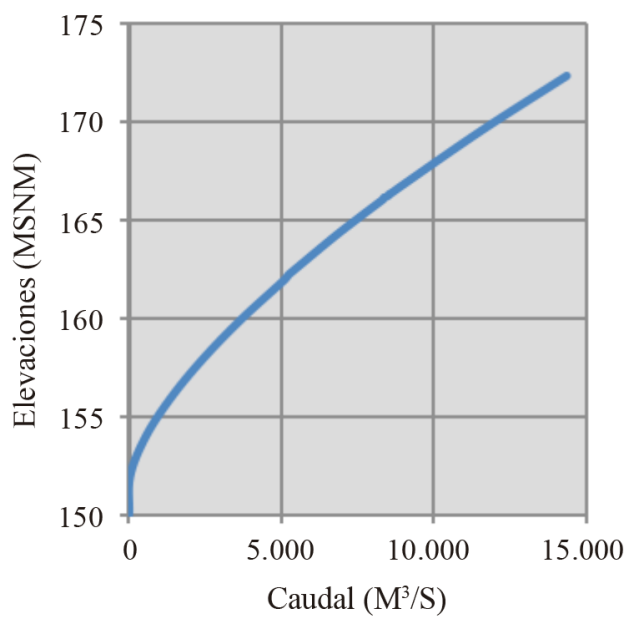

Figura 9. Curva Elevaciones - Caudales.

\section{Escenario 1 - Nivel Inicial 162,35 msnm (NAMO)} Se realizaron las simulaciones correspondientes al tránsito de avenidas; a continuación se presentan los resultados de la simulación realizada para el caso de la avenida 10.000 años de período de retorno (Figuras 10 y 11).

De las Figuras 10 y 11 se puede apreciar que para avenidas con períodos de retorno hasta de 10.000 años no se alcanzaría el NAME (Nivel de Aguas Máximas Extraordinarias) de la presa; la máxima elevación alcanzada sería de 166,57 msnm por lo que el nivel alcanzado quedaría $0,09 \mathrm{~m}$ por debajo del NAME (166,66 msnm). 
El análisis descrito se realizó del mismo modo para cada una de las avenidas asociadas a diversos periodos de retorno, los resultados se presentan en la Tabla 9. Por otra parte, en la Figura 12 se grafican los períodos de retorno (años) con respecto a las elevaciones máximas alcanzadas en el embalse, para mostrar la tendencia de los resultados.

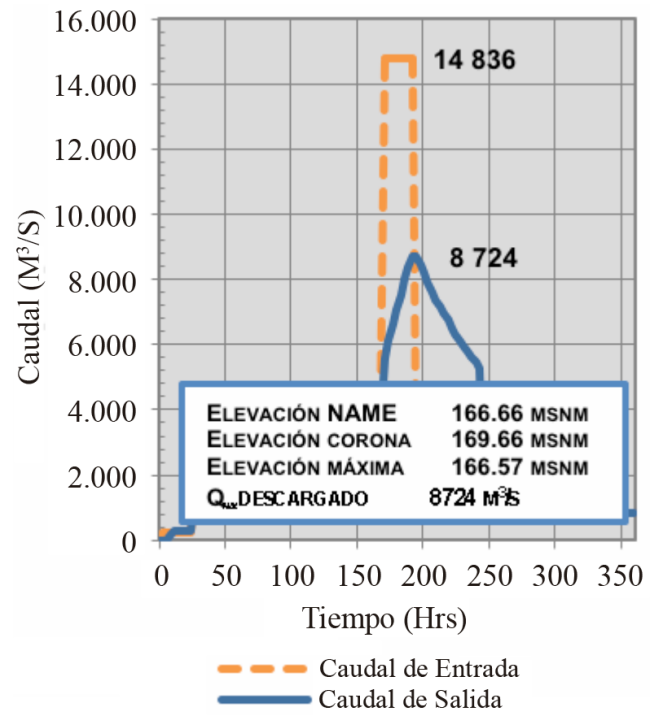

Figura 10. Tránsito de la avenida. Elevación inicial $h=162,35 \mathrm{msnm} . T_{r}=10.000$ años.

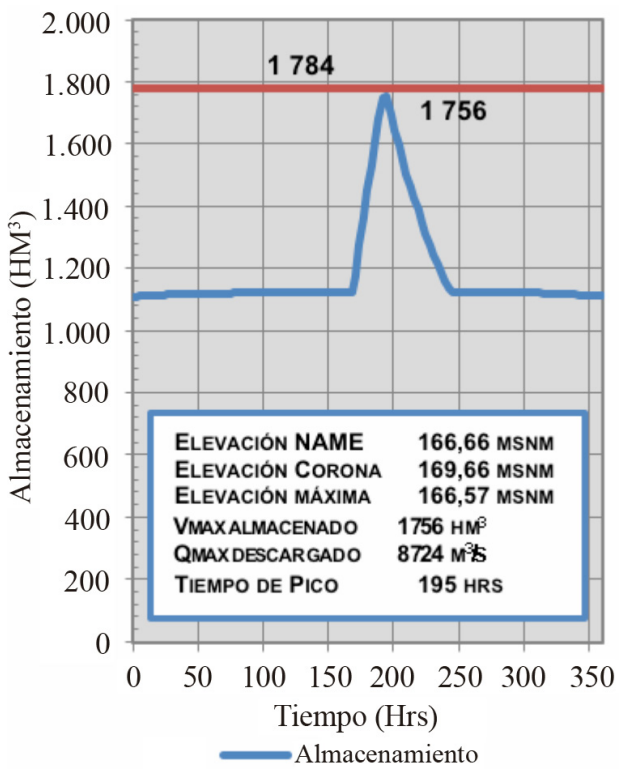

Figura 11. Almacenamiento durante el tránsito de la avenida. Elevación inicial $h=162,35$ msnm. $T_{r}=10.000$ años.
Tabla 9. Resumen del tránsito de avenidas.

\begin{tabular}{|c|c|c|c|c|}
\hline Condición & $\begin{array}{c}\text { Caudal } \\
\text { máximo } \\
\text { de } \\
\text { entrada }\end{array}$ & $\begin{array}{c}\text { Caudal } \\
\text { máximo } \\
\text { de salida }\end{array}$ & $\begin{array}{c}\text { Volumen } \\
\text { máximo }\end{array}$ & $\begin{array}{c}\text { Nivel } \\
\text { máximo } \\
\text { en el } \\
\text { embalse }\end{array}$ \\
\hline $\boldsymbol{T}_{\boldsymbol{r}}$ (Años) & $\mathbf{m}^{\mathbf{3}} / \mathbf{s}$ & $\mathbf{m}^{\mathbf{3}} \mathbf{s}$ & $\mathbf{h m}^{\mathbf{3}}$ & $\mathbf{m}$ \\
\hline 2 & 465,25 & 497,83 & $1.126,46$ & 162,35 \\
\hline 5 & $1.047,02$ & $1.126,83$ & $1.127,26$ & 162,36 \\
\hline 10 & $2.176,78$ & $2.353,41$ & $1.128,81$ & 162,37 \\
\hline 20 & $3.885,76$ & $4.236,84$ & $1.131,20$ & 162,39 \\
\hline 50 & $5.688,69$ & $5.454,17$ & $1.156,76$ & 162,58 \\
\hline 100 & $6.943,35$ & $5.894,00$ & $1.235,10$ & 163,14 \\
\hline 200 & $8.162,49$ & $6.340,43$ & $1.314,62$ & 163,72 \\
\hline 500 & $9.748,29$ & $6.899,69$ & $1.418,27$ & 164,42 \\
\hline 1.000 & $10.949,10$ & $7.309,02$ & $1.497,29$ & 164,91 \\
\hline 2.000 & $12.122,41$ & $7.773,08$ & $1.574,41$ & 165,46 \\
\hline 5.000 & $13.735,71$ & $8.364,95$ & $1.680,56$ & 166,15 \\
\hline 10.000 & $14.835,69$ & $8.723,75$ & $1.756,21$ & 166,57 \\
\hline
\end{tabular}

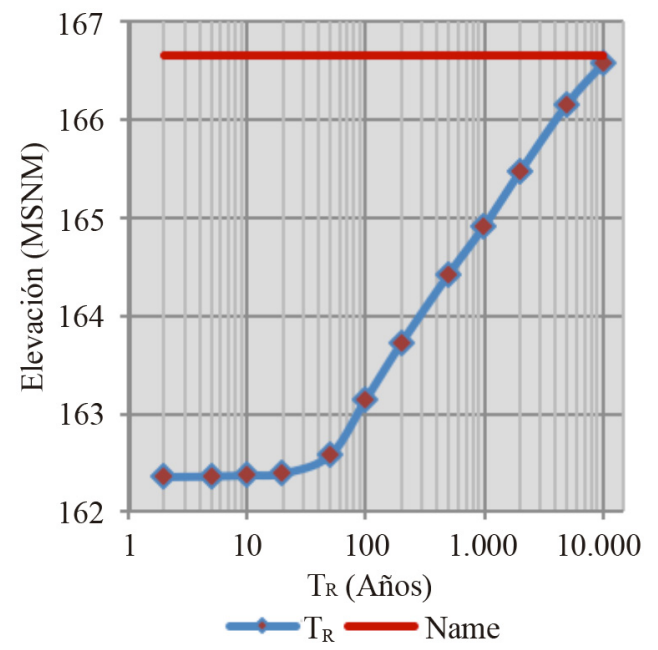

Figura 12. Curva $T_{r}$ - Elevaciones.

\section{Escenario 2 - Niveles medios más frecuentes en el embalse}

Se realizó el análisis de las avenidas simulando su tránsito partiendo de diversos niveles iniciales en el embalse, para ello se empleó el histograma de niveles (Figura 7) obtenido de la optimización de la política de operación del embalse. De dicho histograma se obtuvieron como resultado cuatro niveles más frecuentes en el embalse (Tabla 10), cuyas frecuencias relativas corresponderán a las probabilidades de ocurrencia $\left(P_{\text {Elev }}\right)$ a ser analizadas. 
Tabla 10. Frecuencias de niveles en el embalse.

\begin{tabular}{|c|c|c|c|}
\hline \multicolumn{2}{|c|}{ Intervalos @ 2,0 m } & $\begin{array}{c}\text { Frecuencias } \\
\text { absolutas }\end{array}$ & $\begin{array}{c}\text { Frecuencias } \\
\text { relativas }\end{array}$ \\
\hline 154,0 & 156,0 & 12,00 & 0,115 \\
\hline 156,0 & 158,0 & 19,00 & 0,183 \\
\hline 158,0 & 160,0 & 36,00 & 0,346 \\
\hline 160,0 & 162,0 & 37,00 & 0,356 \\
\hline \multicolumn{2}{|c|}{ Suma } & 104 & 1 \\
\hline
\end{tabular}

Empleando los niveles iniciales definidos en la Tabla 10 y las avenidas de diseño presentadas en la Figura 5, se realizaron los tránsitos de las mismas por el embalse, como se describe a continuación:

\section{Nivel inicial 156 msnm}

Partiendo de la elevación inicial a la cota 156 msnm, se realizaron las simulaciones del tránsito de las avenidas para los períodos de retorno desde 2 hasta 10.000 años, determinando su caudal máximo de descarga, volumen máximo en el embalse, así como la elevación máxima alcanzada (Tabla 11) para definir la probabilidad de excedencia de los caudales máximos de descarga considerando valores de 1.000 a $8.000 \mathrm{~m}^{3} / \mathrm{s}$, a cada $1000 \mathrm{~m}^{3} / \mathrm{s}$, se realizó una interpolación entre los resultados obtenidos, asignándoles su correspondiente periodo de retorno, como se muestra en la Tabla 11 y la Figura 13.

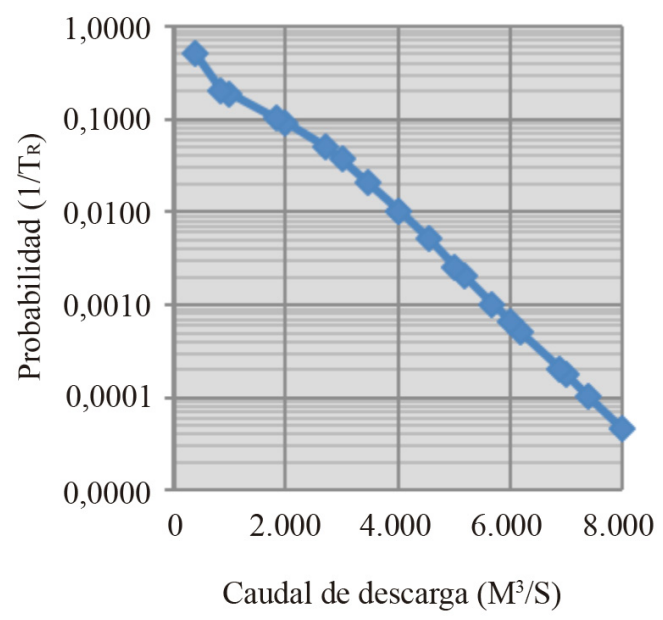

Figura 13. Curva caudal máximo de descarga vs. Probabilidad de excedencia - Nivel inicial $156 \mathrm{msnm}$.
Tabla 11. Resultados de los tránsitos de avenida Nivel inicial $156 \mathrm{msnm}$.

\begin{tabular}{|r|c|c|c|}
\hline \multicolumn{1}{|c|}{$\boldsymbol{T}_{\boldsymbol{r}}$} & $\mathbf{P}$ & $\boldsymbol{Q}_{\text {max Ent }}$ & $\boldsymbol{Q}_{\text {max desc }}$ \\
\hline años & $\mathbf{1} / \boldsymbol{T}_{\boldsymbol{r}}$ & $\mathbf{~ m}^{\mathbf{3}} / \mathbf{s}$ & $\mathbf{~ m}^{\mathbf{3}} \mathbf{s}$ \\
\hline 22.208 & 0,0000 & & $8.000,00$ \\
\hline 10.000 & 0,0001 & $14.835,69$ & $7.400,53$ \\
\hline 5.813 & 0,0002 & & $7.000,00$ \\
\hline 5.000 & 0,0002 & $13.735,71$ & $6.874,14$ \\
\hline 2.000 & 0,0005 & $12.122,41$ & $6.180,57$ \\
\hline 1.522 & 0,0007 & & $6.000,00$ \\
\hline 1.000 & 0,0010 & $10.949,10$ & $5.677,09$ \\
\hline 500 & 0,0020 & $9.748,29$ & $5.182,79$ \\
\hline 398 & 0,0025 & & $5.000,00$ \\
\hline 200 & 0,0050 & $8.162,49$ & $4.544,10$ \\
\hline 100 & 0,0100 & $6.943,35$ & $4.006,15$ \\
\hline 99 & 0,0101 & & $4.000,00$ \\
\hline 50 & 0,0200 & $5.688,69$ & $3.461,48$ \\
\hline 27 & 0,0366 & & $3.000,00$ \\
\hline 20 & 0,0500 & $3.885,76$ & $2.700,09$ \\
\hline 11 & 0,0907 & & $2.000,00$ \\
\hline 10 & 0,1000 & $2.176,78$ & $1.839,74$ \\
\hline 5 & 0,1848 & & $1.000,00$ \\
\hline 5 & 0,2000 & $1.047,02$ & 849,45 \\
\hline 2 & 0,5000 & 465,25 & 376,24 \\
\hline
\end{tabular}

\begin{tabular}{|r|c|c|c|}
\hline \multicolumn{1}{|c|}{$\boldsymbol{T r}$} & $\boldsymbol{P}$ & Vol $_{\text {max }}$ & Nivel $_{\text {max }}$ \\
\hline años & $\mathbf{1} \boldsymbol{T}_{\boldsymbol{r}}$ & $\mathbf{h m}^{\mathbf{3}}$ & $\boldsymbol{m}$ \\
\hline 22.208 & 0,0000 & & \\
\hline 10.000 & 0,0001 & $1.514,95$ & 165,02 \\
\hline 5.813 & 0,0002 & & \\
\hline 5.000 & 0,0002 & $1.413,34$ & 164,39 \\
\hline 2.000 & 0,0005 & $1.286,14$ & 163,51 \\
\hline 1.522 & 0,0007 & & \\
\hline 1.000 & 0,0010 & $1.196,46$ & 162,86 \\
\hline 500 & 0,0020 & $1.105,66$ & 162,20 \\
\hline 398 & 0,0025 & & \\
\hline 200 & 0,0050 & 988,72 & 161,25 \\
\hline 100 & 0,0100 & 900,58 & 160,48 \\
\hline 99 & 0,0101 & & \\
\hline 50 & 0,0200 & 812,68 & 159,67 \\
\hline 27 & 0,0366 & & \\
\hline 20 & 0,0500 & 692,93 & 158,45 \\
\hline 11 & 0,0907 & & \\
\hline 10 & 0,1000 & 609,65 & 157,52 \\
\hline 5 & 0,1848 & & \\
\hline 5 & 0,2000 & 543,14 & 156,70 \\
\hline 2 & 0,5000 & 511,35 & 156,31 \\
\hline
\end{tabular}

El procedimiento descrito se aplicó considerando los niveles iniciales a las cotas 158, 160 y $162 \mathrm{msnm}$. 
Resumen general de los resultados obtenidos para diversos niveles iniciales

A continuación, en la Figura 14 se presenta el análisis gráfico de las distribuciones de probabilidad de los caudales máximos de descarga para los diversos niveles iniciales estudiados:

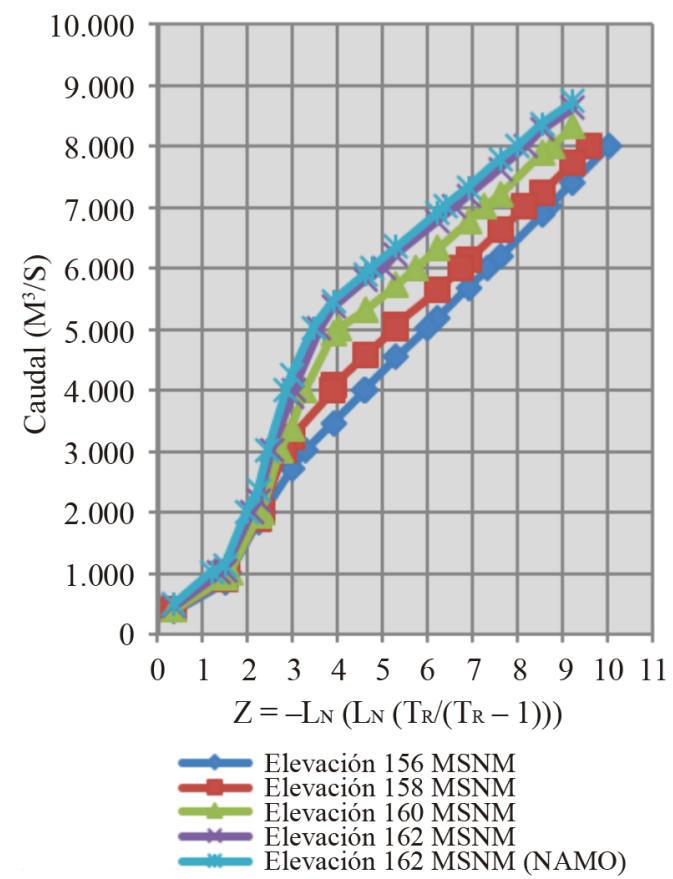

Figura 14. Resumen general de los caudales asociados a diversos niveles iniciales.

\section{Análisis de la probabilidad de excedencia conjunta}

En el escenario número dos se describieron los resultados obtenidos del tránsito de las avenidas ante diversos niveles iniciales. Al considerar las probabilidades de excedencias obtenidas para cada caudal de descarga con la probabilidad de ocurrencia correspondiente a cada elevación inicial $\left(P_{\text {Elev }}\right)$, se determinó la probabilidad de excedencia conjunta $\left(P_{T}\right)$.

Para ello, se realizó, para cada caudal entre 1.000 y $8.000 \mathrm{~m}^{3} / \mathrm{s}$ la suma del producto de la probabilidad asociada a cada nivel inicial $\left(P_{\text {Elev }}\right)$ por la probabilidad de excedencia del caudal correspondiente $\left(P_{Q / e l e v}\right)$, es decir:

$$
P_{T i}=\sum_{k=156}^{162} P_{Q_{i / k}} P_{\text {Elev }_{k}}
$$

Donde $P_{T i}$ es la probabilidad conjunta de excedencia asociada al caudal máximo de descarga $Q_{i} ; P_{\text {Elev }}$ es la probabilidad de que el tránsito se inicie en la elevación $k$, y $P_{Q_{i / k}}$ la probabilidad de que el caudal máximo de descarga sea mayor o igual que $Q_{i}$ dada la elevación inicial $k$.

A continuación, en la Tabla 12, se presentan las probabilidades conjuntas obtenidas para cada uno de los caudales analizados:

Tabla 12. Probabilidad de excedencia conjunta.

\begin{tabular}{|c|c|c|c|c|}
\hline Elev. inicial & \multicolumn{2}{|c|}{ Caudal $\left(\mathrm{m}^{3} / \mathrm{s}\right)$} & \multicolumn{2}{|c|}{8.000} \\
\hline $\mathrm{msnm}$ & $T_{r}$ & $P_{Q}$ & $P_{\text {Elev }}$ & $\boldsymbol{P}_{Q} \boldsymbol{P}_{\text {Elev }}$ \\
\hline 156 & 22.208 & 0,00005 & 0,11538 & 0,00001 \\
\hline 158 & 14.156 & 0,00007 & 0,18269 & 0,00001 \\
\hline 160 & 6.252 & 0,00016 & 0,34615 & 0,00006 \\
\hline 162 & 3.527 & 0,00028 & 0,35577 & 0,00010 \\
\hline & & & $P_{T} \mathbf{8 . 0 0 0}$ & 0,00017 \\
\hline Elev. inicial & \multicolumn{2}{|c|}{ Caudal $\left(\mathrm{m}^{3} / \mathrm{s}\right)$} & \multicolumn{2}{|c|}{7.000} \\
\hline $\mathrm{msnm}$ & $T_{r}$ & $P_{Q}$ & $P_{\text {Elev }}$ & $P_{Q} P_{\text {Elev }}$ \\
\hline 156 & 5.813 & 0,00017 & 0,11538 & 0,00002 \\
\hline 158 & 3.433 & 0,00029 & 0,18269 & 0,00005 \\
\hline 160 & 1.373 & 0,00073 & 0,34615 & 0,00025 \\
\hline 162 & 727 & 0,00138 & 0,35577 & 0,00049 \\
\hline & & & $P_{T} 7.000$ & 0,00081 \\
\hline Elev. inicial & \multicolumn{2}{|c|}{ Caudal $\left(\mathrm{m}^{3} / \mathrm{s}\right)$} & \multicolumn{2}{|c|}{6.000} \\
\hline $\mathrm{msnm}$ & $T_{r}$ & $P_{Q}$ & $P_{\text {Elev }}$ & $P_{Q} P_{\text {Elev }}$ \\
\hline 156 & 1.522 & 0,00066 & 0,11538 & 0,00008 \\
\hline 158 & 833 & 0,00120 & 0,18269 & 0,00022 \\
\hline 160 & 301 & 0,00332 & 0,34615 & 0,00115 \\
\hline 162 & 150 & 0,00668 & 0,35577 & 0,00238 \\
\hline & & & $P_{T} 6.000$ & 0,0382 \\
\hline Elev. inicial & \multicolumn{2}{|c|}{ Caudal $\left(\mathrm{m}^{3} / \mathrm{s}\right)$} & \multicolumn{2}{|c|}{5.000} \\
\hline msnm & $T_{r}$ & $P_{Q}$ & $\boldsymbol{P}_{\text {Elev }}$ & $\boldsymbol{P}_{Q} \boldsymbol{P}_{\text {Elev }}$ \\
\hline 156 & 398 & 0,00251 & 0,11538 & 0,00029 \\
\hline 158 & 196 & 0,00510 & 0,18269 & 0,00093 \\
\hline 160 & 56 & 0,01798 & 0,34615 & 0,00622 \\
\hline 162 & 37 & 0,02732 & 0,36000 & 0,00983 \\
\hline & & & $P_{T} 5.000$ & 0,01728 \\
\hline Elev Inicial & \multicolumn{2}{|c|}{ Caudal $\left(\mathrm{m}^{3} / \mathrm{s}\right)$} & \multicolumn{2}{|c|}{4.000} \\
\hline msnm & $T_{r}$ & $P_{Q}$ & $\boldsymbol{P}_{\text {Elev }}$ & $P_{Q} P_{\text {Elev }}$ \\
\hline 156 & 99 & 0,01010 & 0,11538 & 0,00117 \\
\hline 158 & 49 & 0,02042 & 0,18269 & 0,00373 \\
\hline 160 & 26 & 0,03792 & 0,34615 & 0,01313 \\
\hline 162 & 21 & 0,04767 & 0,36000 & 0,01716 \\
\hline & & & $P_{T} 4.000$ & 0,03519 \\
\hline
\end{tabular}




\begin{tabular}{|c|c|c|c|c|}
\hline \multirow{2}{*}{$\begin{array}{c}\text { Elev. inicial } \\
\mathrm{msnm}\end{array}$} & \multicolumn{2}{|c|}{ Caudal $\left(\mathrm{m}^{3} / \mathrm{s}\right)$} & \multicolumn{2}{|c|}{3.000} \\
\hline & $T_{r}$ & $P_{Q}$ & $P_{\text {Elev }}$ & $\boldsymbol{P}_{Q} \boldsymbol{P}_{\text {Elev }}$ \\
\hline 156 & 27 & 0,03664 & 0,11538 & 0,00423 \\
\hline 158 & 17 & 0,05820 & 0,18269 & 0,01063 \\
\hline 160 & 16 & 0,06318 & 0,34615 & 0,02187 \\
\hline 162 & 13 & 0,07591 & 0,36000 & 0,02733 \\
\hline & $P_{T} 3.000$ & 0,06406 \\
\hline Elev. inicial & \multicolumn{2}{|c|}{ Caudal $\left(\mathrm{m}^{3} / \mathrm{s}\right)$} & \multicolumn{2}{|c|}{2000} \\
\hline msnm & $T_{r}$ & $P_{Q}$ & $\boldsymbol{P}_{\text {Elev }}$ & $\boldsymbol{P}_{Q} \boldsymbol{P}_{\text {Elev }}$ \\
\hline 156 & 11 & 0,09069 & 0,11538 & 0,01046 \\
\hline 158 & 11 & 0,09500 & 0,18269 & 0,01736 \\
\hline 160 & 11 & 0,09500 & 0,34615 & 0,03288 \\
\hline \multirow[t]{2}{*}{162} & 9 & 0,11565 & 0,36000 & 0,04163 \\
\hline & & & $P_{T} 2.000$ & 0,10234 \\
\hline Elev. inicial & \multicolumn{2}{|c|}{ Caudal $\left(\mathrm{m}^{3} / \mathrm{s}\right)$} & \multicolumn{2}{|c|}{1.000} \\
\hline msnm & $T_{r}$ & $P_{Q}$ & $P_{\text {Elev }}$ & $\boldsymbol{P}_{Q} \boldsymbol{P}_{\text {Elev }}$ \\
\hline 156 & 5 & 0,18480 & 0,11538 & 0,02132 \\
\hline 158 & 5 & 0,18989 & 0,18269 & 0,03469 \\
\hline 160 & 6 & 0,18000 & 0,34615 & 0,06231 \\
\hline 162 & 4 & 0,22425 & 0,36000 & 0,08073 \\
\hline & & & $P_{T} 1.000$ & 0,19905 \\
\hline
\end{tabular}

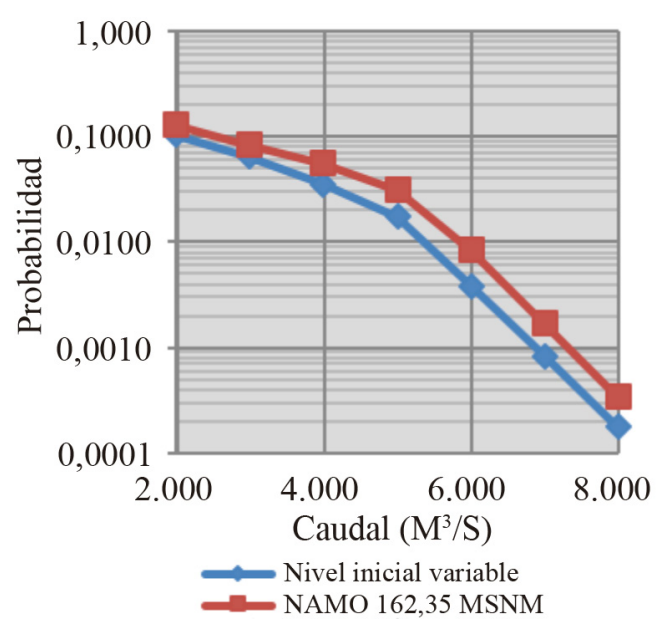

Figura 15. Resumen Probabilidades de excedencia conjunta. Presa El Cuchillo.

Del análisis realizado se elaboró la Tabla 13 y la Figura 15 en donde se muestra la tendencia de la probabilidad de excedencia conjunta asociada a cada caudal máximo de descarga, comparada con la obtenida considerando el nivel inicial a la elevación del NAMO.
Tabla 13. Resumen Probabilidad de excedencia conjunta.

\begin{tabular}{|c|c|c|}
\hline Caudal, $\mathbf{~ m}^{\mathbf{3} / \mathbf{s}}$ & $\boldsymbol{P}_{\boldsymbol{Q}} \boldsymbol{P}_{\text {Elev }}$ & $\boldsymbol{P}_{\boldsymbol{Q}} \mathbf{N A M O}$ \\
\hline 1.000 & 0,19905 & 0,26049 \\
\hline 2.000 & 0,10234 & 0,12881 \\
\hline 3.000 & 0,06406 & 0,08283 \\
\hline 4.000 & 0,03519 & 0,05629 \\
\hline 5.000 & 0,01728 & 0,03119 \\
\hline 6.000 & 0,00382 & 0,00846 \\
\hline 7.000 & 0,00081 & 0,00169 \\
\hline 8.000 & 0,00017 & 0,00034 \\
\hline
\end{tabular}

\section{Tránsito hidráulico por el cauce}

Considerando los caudales de salida utilizados en el análisis se procedió a simular su tránsito por el cauce. Para ello, se empleó el modelo digital de elevaciones de la página de ASTER GDEM [25] (ASTER Global Digital Elevation Model), realizando el análisis en el área comprendida entre el vertedor de la presa El Cuchillo hasta el embalse de la presa ubicada aguas abajo (Marte R. Gómez). Dicha área fue delimitada empleando la plataforma Global Mapper ${ }^{\circledR}[26]$.

Para llevar a cabo la simulación del tránsito de la avenida por el cauce, el modelo digital de elevaciones fue exportado al programa HEC - RAS ® [27], considerando los distintos caudales analizados.

Los resultados obtenidos de la simulación de HEC RAS, se incorporaron al programa LAMINA $₫$ [28], en el que a partir de los niveles del agua obtenidos por cada sección del cauce determina el área o superficie inundada. En la Tabla 14, se presentan las áreas de afectación asociadas a su correspondiente probabilidad de excedencia para los dos escenarios considerados.

\section{Costos por afectación}

Partiendo de costos por hectárea de afectación obtenidos en el Proyecto Hidroeléctrico Chicoasén II (90.000 pesos en el año 2014), se consideró prudente emplear un índice de inflación media del $1 \%$ al 2015, correspondiendo a un costo por hectárea de afectación de alrededor de 90.900 pesos; dicho costo fue multiplicado por las áreas de afectación (Tabla 14), determinando los costos asociados a diversas probabilidades de excedencia (Figura 16). 
Tabla 14. Costo por área de afectación.

\begin{tabular}{|c|c|c|c|}
\hline \multicolumn{4}{|c|}{ Nivel de inicio variable } \\
\hline$\underset{\mathbf{m}^{3} / \mathbf{s}}{Q}$ & $\begin{array}{c}\text { Área } \\
\text { ha }\end{array}$ & $\begin{array}{c}\text { Costo } \\
\text { Mill. de pesos }\end{array}$ & $\begin{array}{c}P_{f} \\
1 / T_{r}\end{array}$ \\
\hline 1.000 & 0,00 & 0,00 & 0,1991 \\
\hline 2.000 & 409,24 & 36,83 & 0,1023 \\
\hline 3.000 & 639,53 & 57,56 & 0,0641 \\
\hline 4.000 & 787,94 & 70,92 & 0,0352 \\
\hline 5.000 & 876,30 & 78,87 & 0,0173 \\
\hline 6.000 & 933,82 & 84,04 & 0,0038 \\
\hline 7.000 & 981,59 & 88,34 & 0,0008 \\
\hline 8.000 & $1.011,04$ & 90,99 & 0,0002 \\
\hline \multicolumn{4}{|c|}{ NAMO } \\
\hline$\underset{\mathbf{m}^{3} / \mathbf{s}}{Q}$ & $\begin{array}{c}\text { Área } \\
\text { ha }\end{array}$ & $\begin{array}{c}\text { Costo } \\
\text { Mill de pesos }\end{array}$ & $\begin{array}{c}P_{f} \\
1 / T_{r}\end{array}$ \\
\hline 1.000 & 0,00 & 0,00 & 0,2605 \\
\hline 2.000 & 409,24 & 36,83 & 0,1288 \\
\hline 3.000 & 639,53 & 57,56 & 0,0828 \\
\hline 4.000 & 787,94 & 70,92 & 0,0563 \\
\hline 5.000 & 876,30 & 78,87 & 0,0312 \\
\hline 6.000 & 933,82 & 84,04 & 0,0085 \\
\hline 7.000 & 981,59 & 88,34 & 0,0017 \\
\hline 8.000 & $1.011,04$ & 90,99 & 0,0003 \\
\hline
\end{tabular}

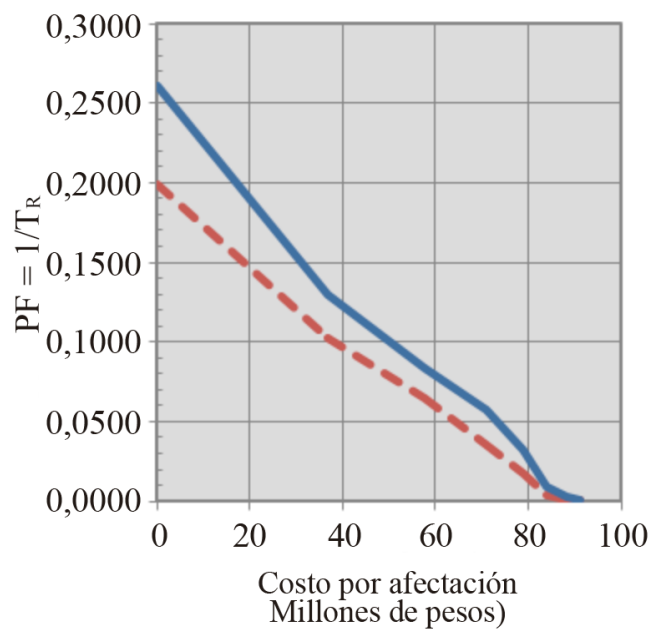

- Nivel inicial variable NAMO

Figura 16. Costos asociados a diversas probabilidades de excedencia.

De la Figura 16, el costo esperado se determina como el área bajo las curvas graficadas, cuyo valor
Tabla 15. Costo total de riesgo esperado.

\begin{tabular}{|l|c|}
\hline \multicolumn{1}{|c|}{ Análisis } & Millones de pesos \\
\hline Nivel inicial variable & 8,20 \\
\hline NAMO & 10,74 \\
\hline
\end{tabular}

se presenta en la Tabla 15, en la que se comparan los dos escenarios estudiados.

\section{CONCLUSIONES}

La estimación del riesgo por inundación aguas abajo de una presa se hace tradicionalmente suponiendo que las avenidas de diseño ingresan cuando el nivel en el vaso es el del NAMO, lo que implica una sobrestimación de dicho riesgo. En este trabajo se propone un método que permite obtener una estimación más completa del riesgo de inundaciones aguas abajo de una presa, el que se aplica a la zona situada aguas abajo de la presa El Cuchillo.

El desarrollo del método incluye el cálculo de las avenidas de diseño para diferentes períodos de retorno, la definición de la política de operación a largo plazo que hace óptima una función objetivo, el tránsito de las avenidas de diseño -primero por el vaso de la presa suponiendo distintas elevaciones iniciales y luego por el río-, y la estimación de los daños.

La metodología propuesta optimiza y/o mejora la operación de los embalses en diversos puntos, por ejemplo, permite garantizar la confiabilidad de los registros (ya sean horarios, diarios o mensuales).

Como parte del resultado del funcionamiento analítico de vaso optimizado, se obtienen los niveles más frecuentes en el embalse (histograma de elevaciones medias), los que serán las condiciones de inicio en el tránsito de las avenidas de diseño generando un escenario de análisis que parte de niveles asociados a su probabilidad de ocurrencia $\left(P_{\text {Elev }}\right)$.

Por otra parte, del tránsito de las avenidas de diseño partiendo de diferentes elevaciones iniciales, se obtuvieron las probabilidades de excedencia $\left(P_{Q}\right)$, detallando si la obra de excedencias cuenta con la capacidad de descarga necesaria ante la presencia de avenidas máximas sin que el NAME sea sobrepasado. 
Lo anterior, permite garantizar la seguridad integral de las obras, guiando al operador a mantener el embalse por debajo un determinado nivel (curva guía), o en su caso, determinando la inclusión de una obra de excedencias auxiliar para preservar el NAME no sea sobrepasado.

La presente metodología define la probabilidad de que una gama de eventos se llegasen a presentar, considerando como otra variable la elevación inicial en el embalse. Lo anterior involucra una condición realista en la presa, ya que convencionalmente se supone que ante cualquier evento la elevación en el embalse es el NAMO.

Se puede concluir, que al emplear la metodología propuesta, se refleja un ahorro sustancial en los daños esperados, al comparar los costos obtenidos bajo un nivel inicial variable con respecto a un análisis convencional realizado bajo el NAMO, en donde el último sobreestima los costos.

Para el caso de la presa El Cuchillo la avenida de diseño original ( $\operatorname{Tr} 10000$ años) considera un caudal máximo de entrada de $15.000 \mathrm{~m}^{3} / \mathrm{s}$, valor que se modificó muy ligeramente (a $14.836 \mathrm{~m}^{3} / \mathrm{s}$ ) con los datos actualizados.

La optimización de la política de operación en embalse, permite operar con los niveles en la presa por arriba de los históricos, incrementando el volumen útil en el embalse sin superar la cota del NAMO. Al simular el funcionamiento de la presa con los ingresos históricos se estimaron las frecuencias de las elevaciones en el embalse para la época de crecientes.

Del simular el tránsito de las avenidas de diseño, partiendo de diferentes elevaciones iniciales, se obtuvieron las probabilidades de excedencia conjunta ecuación (5) asociadas a los caudales máximos de descarga.

Como se puede apreciar en la Figura 16 y en la Tabla 15, el procedimiento propuesto, que considera los diversos niveles iniciales en el embalse, permite una estimación más precisa del riesgo de inundación aguas abajo de la presa, mientras que el procedimiento tradicional (considerando un nivel inicial a la cota del NAMO) sobreestima los costos del daño por inundación.

\section{REFERENCIAS}

[1] R. Rebolledo S. "Operación óptima de un sistema hidráulico formado por dos presas en paralelo". UNAM. 1990. URL: http:// bibliotecacentral.unam.mx/

[2] R. Avilés H. “Optimización en línea de presas hidroeléctricas”. UNAM, 1994. URL: http:// bibliotecacentral.unam.mx/

[3] C. Contreras C. "Operación óptima de sistemas de presas en cascada. Aplicaciones al sistema del río Grijalva”. UNAM. 1999. URL: http://bibliotecacentral.unam.mx/

[4] M. Arganis J. "Operación óptima de un sistema de presas en cascada para generación hidroeléctrica tomando en cuenta condiciones reales de operación y el uso de muestras sintéticas para el pronóstico". UNAM. 2004. URL: http:// bibliotecacentral.unam.mx/

[5] E. Sánchez C. y A. Wagner G. "Determinación de reglas de operación óptima para dos embalses, utilizando un algoritmo genético. XIII Escuela Nacional de Optimización y Análisis Numérico". Universidad Autónoma de Coahuila, Sociedad Matemática Mexicana. Saltillo, Coahuila, México. 2003.

[6] E. Sánchez C. y A. Wagner G. "Modelo numérico para la operación óptima de un hidrosistema de aguas superficiales". Instituto Mexicano de Tecnología del Agua. Morelos, México. 2004.

[7] R. Domínguez M., R. Mendoza R., A. Alvarado C. y E. Márquez L. "Operación integral del sistema hidroeléctrico del río Grijalva”. CFE. Instituto de Ingeniería. UNAM. 1993.

[8] R. Domínguez M., R. Mendoza R. y C. Contreras C. "Políticas de operación del sistema de presas". CNA. Instituto de Ingeniería. UNAM. 1998.

[9] R. Domínguez M. y R. Mendoza R., "Funcionamiento de las Presas Angostura, Chicoasén, Malpaso y Peñitas sobre el Río Grijalva”. CNA. Instituto de Ingeniería. UNAM. 2000.

[10] R. Domínguez M., R. Mendoza R. y M. Arganis J. "Revisión de políticas de operación quincenal de las presas Angostura y Malpaso, en el Río Grijalva”. CFE. Instituto de Ingeniería. UNAM. 2001. 
[11] R. Domínguez M., L.J.M. Arganis, E. Carrizosa E., G.E. Fuentes M. y C.A. Echeverría V. "Determinación de avenidas de diseño y ajuste de los parámetros del modelo de optimización de las políticas de operación del sistema de presas del río Grijalva”. CFE. Instituto de Ingeniería. UNAM. 2006.

[12] R. Dittmann, F. Froehlich F., Pohl R. and M. Ostrowski. "A management system for operating rules of multipurpose reservoirs allowing for both extreme floods and ecological". 4th International Symposium on Flood Defence. 2008.

[13] J. Vigyan B. "Development of operation policy for Tawa dam". National Institute of Hydrology. Roorkee, India. 1997.

[14] N. Pradhan S. and K. Tripathy U. "Optimization of the Operating Policy of The Multipurpose Hirakud Reservoir by Genetic Algorithm". American Journal of Engineering Research. 2013.

[15] M. Jiménez E. "Programa AX". Área de Riesgos Hidrometeorológicos. Centro Nacional de Prevención de Desastres. México. 1996.

[16] R. Domínguez, M. and Carlóz G.T. "Análisis Estadístico, Capítulo A.1.6 del Manual de Diseño de Obras Civiles". CFE, México. 1981.

[17] E. Gumbel. "Statistics of Extremes". New York, USA. Columbia University Press. 1958.

[18] R. Domínguez M., G.E. Fuentes M. y M.L. Arganis J. "Optimización de los Parámetros de la Función Doble Gumbel Usando Algoritmos Genéticos en una Serie de Caudales Máximos Anuales". XXI Congreso Latinoamericano de Hidráulica, pp. B59, 1-9. Sao Paulo, Brasil. 2004.
[19] Comisión Federal de Electricidad. "Manual de Diseño de Obras Civiles Capítulo A.1.10. Avenida de Diseño". 1980.

[20] L. Chang and F. Chang. "Multi-objective evolutionary algorithm for operating parallel reservoir system". Journal of Hydrology. Vol. 377, pp. 12-20. 2009.

[21] Y. Mathur and S. Nikam. "Optimal reservoir operation policies using genetic algorithm". International Journal of Engineering \& Technology. Vol. 1, pp. 184-187. 2009.

[22] J.T. Kuo, W.C. Cheng, L. Chen L. "Multi objective water resources systems analysis using genetic algorithms - application to Chou-shui river basin, Taiwan". Water Sci Technology Vol. 48, pp. 71-77. 2003.

[23] R.R. Mendoza. "Manual del Programa de Optimización vía Programación Dinámica Estocástica”. 2010.

[24] H. Marengo M., F.J. Contreras W. y J.C. Rivero A. "Tránsito de una avenida por un vaso”. CFE. 2005.

[25] "Advanced Spaceborne Thermal Emission and Reflection Radiometer Globe Digital Elevation Model (ASTER GDEM)”. 2014. URL: http://gdem.ersdac.jspacesystems. or.jp/

[26] Blue Marble Geographics. Global Mapper. URL: http://www.bluemarblegeo.com/ products/global-mapper.php

[27] US Army Corps of Engineers. "Hydrologic Engineering Centers River Analysis System (HEC-RAS)". 2014. URL: http://www.hec. usace.army.mil/software/hec-ras

[28] GITS. "Grupo de Investigación en Transporte de Sedimentos". LAMINA. 2014. URL: http://www.gits.ws/ 